$1-1-1977$

\title{
Tables and procedures for estimating weights of some Appalachian hardwoods
}

Harry V. Wiant

Follow this and additional works at: https://researchrepository.wvu.edu/ wv_agricultural_and_forestry_experiment_station_bulletins

\section{Digital Commons Citation}

Wiant, Harry V., "Tables and procedures for estimating weights of some Appalachian hardwoods" (1977). West Virginia Agricultural and Forestry Experiment Station Bulletins. 659.

https://researchrepository.wvu.edu/wv_agricultural_and_forestry_experiment_station_bulletins/560 @ WVU. It has been accepted for inclusion in West Virginia Agricultural and Forestry Experiment Station Bulletins by an authorized administrator of The Research Repository @ WVU. For more information, please contact ian.harmon@mail.wvu.edu. 


\section{Tables and Procedures for Estimating Weights of Some Appalachian Hardwoods}

Bulletin 659T

December 1977

West Virginia University

Agricultural and Forestry Experiment Station

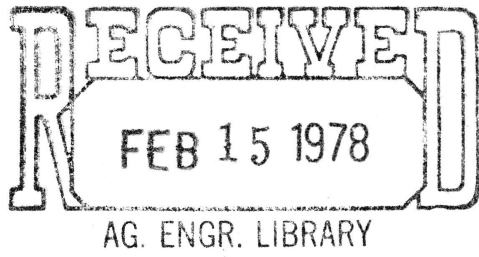

WEST VIRGINIA UNIVERSITY
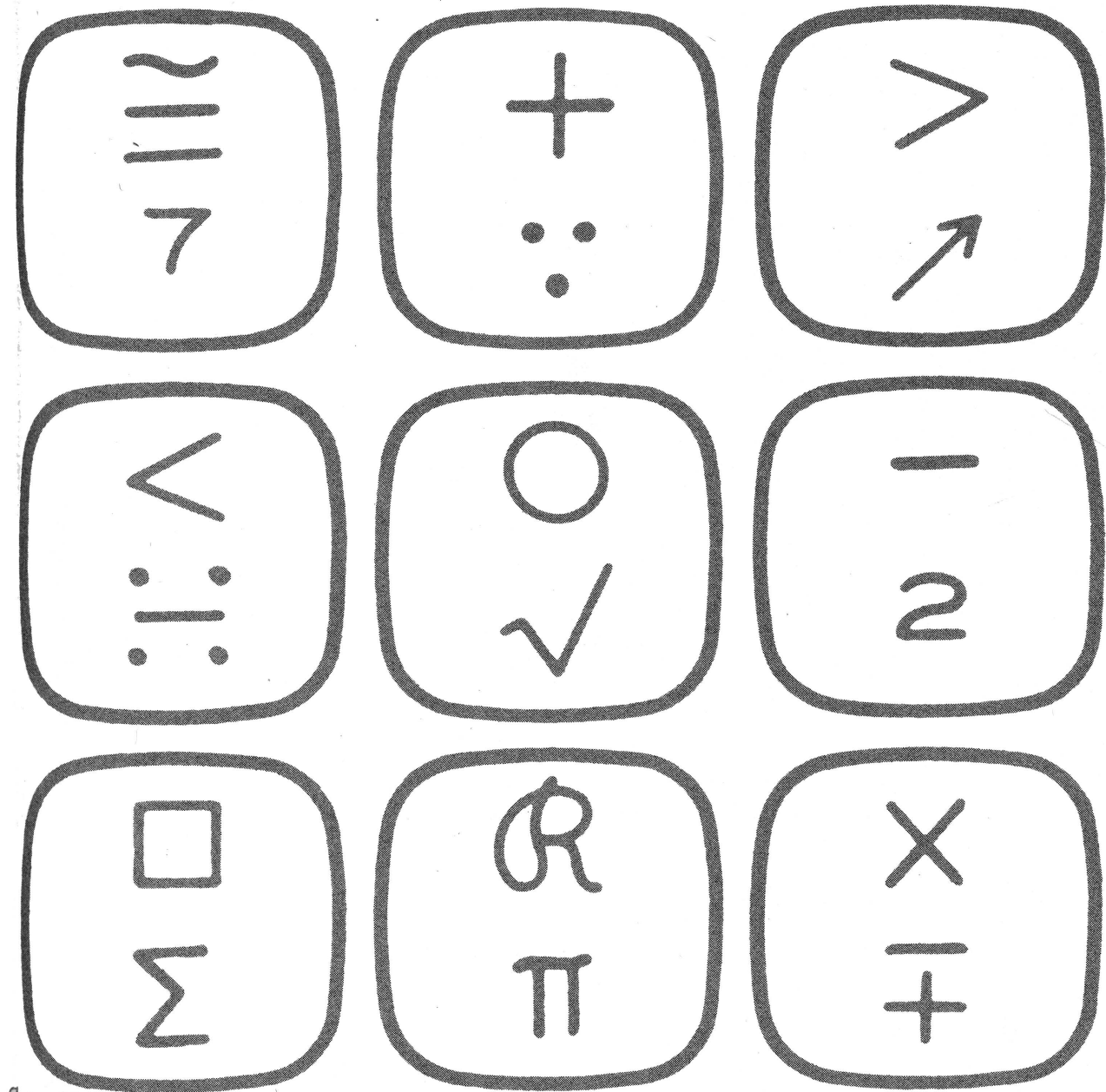

$\$ 127$

.E

no.659 T 


\section{AUTHORS}

Harry V. Wiant, Jr. is Forest Scientist in the West Virginia University Agricultural and Forestry Experiment Station. Junior authors were graduate students in the Division of Forestry, West Virginia University.

\section{ACKNOWLEDGMENTS}

Appreciation is extended to the Westvaco Corporation, and especially Mr. Bruce B. Brenneman, Research Center Leader at Rupert, West Virginia, for their cooperation and review of the manuscript and to the West Virginia Department of Natural Resources for the use of the West Virginia University Forest. Thanks are expressed to others who reviewed the manuscript, including Dr. Harold $E$. Young, University of Maine; Mr. Jeffrey L. Wartluft, USDA Forest Service; and Professor William R. Maxey, West Virginia University

West Virginia University

Agricultural and Forestry Experiment Station

College of Agriculture and Forestry

Dale W. Zinn, Director

Morgantown 


\section{PREFACE}

The weight tables in this publication, while based on data collected in northern West Virginia, may prove useful in other areas of the Appalachian region; however, they should be adequately tested before they are utilized elsewhere. The sampling procedures described are of more general utility. 
Blank Page in Original Bulletin 


\section{Contents}

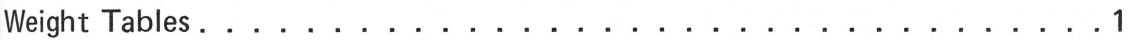

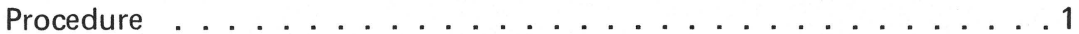

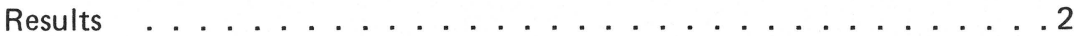

Field Estimates of Weight . . . . . . . . . . . . . 2

Weight Estimates When Marking Timber . . . . . . . . . 15

Weight Estimates Using Fixed-Area Plots . . . . . . . . . . . 15

Weight Estimates Using Point Samples . . . . . . . . . . . . 17

Weight Estimates Using Point and 3P Sampling . . . . . . . . . . . 18

Computerized Approach . . . . . . . . . . . . . . . 21

Combined Equations for Red and White Oaks . . . . . . . . . . 22

Selected References . . . . . . . . . . . . . . . 36

\section{TABLES}

1. Total tree green weight $\ldots \ldots \ldots \ldots \ldots \ldots$

2. Total tree dry weight . . . . . . . . . . . .

3. Total tree dry weight without bark . . . . . . . . . . 5

4. Total tree dry weight of bark . . . . . . . . . . . . 6

5. Green weight of branches . . . . . . . . . . . . 7

6. Dry weight of branches . . . . . . . . . . . . 8

7. Dry weight of branches without bark . . . . . . . . 9

8. Dry weight of branch bark . . . . . . . . . . . 10

9. Green weight to a 4 -inch dob . . . . . . . . . . 11

10. Dry weight to a 4 -inch dob . . . . . . . . . . . 12

11. Dry weight without bark to a 4 -inch dob . . . . . . . 13

12. Dry weight of bark to a 4 -inch dob . . . . . . . . . 14

13. Total tree green weight per acre per in-tree $(B A F=10) \ldots \ldots 23$

14. Total tree dry weight per acre rer in-tree $(B A F=10) \quad \ldots \ldots . .24$

15. Total tree dry weight without bark per acre per in-tree $(B A F=10) \ldots 25$

16. Total tree dry weight of bark per acre per in-tree $(B A F=10) \ldots \ldots 26$

17. Green weight of branches per acre per in-tree $(B A F=10) \ldots \ldots 27$

18. Dry weight of branches per acre per in-tree $(B A F=10) \ldots \ldots 28$

19. Dry weight of branches without park per acre per in-tree $(B A F=10) \quad .29$

20. Dry weight of branch bark per acre per in-tree $(B A F=10) \ldots \ldots 30$

21. Green weight to a 4 -inch dob per acre per in-tree $(B A F=10) \ldots 31$

22. Dry weight to a 4-inch dob per acre per in-tree $(B A F=10) \quad \ldots . .32$

23. Dry weight without bark to a 4-inch dob per acre per in-tree $(B A F=10) 33$

24. Dry weight of bark to a 4 -inch dob per acre per in-tree $(B A F=10) \quad \ldots 34$

25 . Weight equations for the red oak and white oak groups . . . . . 35 
Blank Page in Original Bulletin 


\title{
Tables and Procedures for Estimating Weights of \\ Some Appalachian Hardwoods
}

\author{
Harry V. Wiant, Jr., Carter E. Sheetz, Andrew Colaninno, \\ James C. DeMoss, and Froylan Castaneda
}

\section{WEIGHT TABLES}

The introduction of whole-tree chipping operations in West Virginia forests has stimulated interest in the development of weight tables for field use. These tables facilitate the estimation of weight of standing trees to be chipped and eliminate the need to convert from cords or cubic feet to weight. Weight tables were developed for some Appalachian hardwoods in northern West Virginia.

\section{Procedure}

Nineteen to 22 trees, ranging from 2 to 16 inches in diameter at 4.5 feet $(\mathrm{dbh})$, were selected for study on or near the West Virginia University Forest ${ }^{1}$ near Morgantown for each of the following species:

$\begin{array}{ll}\text { Code } & \text { Species } \\ \text { NRO } & \text { northern red oak (Quercus rubra) } \\ \text { BO } & \text { black oak (Q. velutina) } \\ \text { SO } & \text { scarlet oak (Q. coccinea) } \\ \text { WO } & \text { white oak (Q. alba) } \\ \text { CO } & \text { chestnut oak (Q. prinus) } \\ \text { YP } & \text { yellow-poplar (Liriodendron tulipifera) } \\ \text { H } & \text { hickories (Carya spp.) } \\ \text { BC } & \text { black cherry (Prunus serotina) } \\ \text { RM } & \text { red maple (Acer rubrum) }\end{array}$

Trees were felled, sectioned, and weighed in the field, and oven-dried weights were determined from wedge-shaped samples taken at 4-foot bucking points and from branch samples. All stem material less than 4 inches, diameter outside bark (dob), and limbs were considered as branches. Stumps, approximately $1 / 2$-foot, roots, and leaves were not included in this study.

\footnotetext{
${ }^{1}$ The West Virginia University Forest is evenaged, about 45 years old, with an average site index, using Schnur's (1937) curves, of 73.
} 


\section{Results}

Analyses indicated dbh accounted for most of the variation in total ovendry weight. Inclusion of total or merchantable heights did not improve relation. ships appreciably. This indicates the value of local weight tables, using dbh alone, for weight estimations. Tables 1 to 12 give green and dry weights for the various species.

Regression models tested included:

$$
\begin{aligned}
& W=a+b D^{2} \\
& W=a+b D+c D^{2} \\
& W=a D^{b}
\end{aligned}
$$

where: $\quad W=$ weight in pounds

$\mathrm{D}=\mathrm{dbh}$

$\mathrm{a}, \mathrm{b}$, and $\mathrm{c}=$ regression constants

Although there are more sophisticated statistical procedures, models providing the best $R^{2}$-values (or $r^{2}$ ) were accepted and are given with their standard errors and standard errors as a percent of the W-means below each table. It should be noted that the reliability of equations, as indicated by standard errors, was better for total tree weights than for branch material.

If weights for species not included in this study are desired, it may be possible to use values for a closely related species in terms of growth habit, wood density, etc. For example, four trees for each of several minor species, selected to span the dbh-range, indicated total weights of cucumbertree (Magnolia acuminata) and bigtooth aspen (Populus grandidentata) are approximated by yellow-poplar; and sweet birch (Betula lenta) by black cherry.

\section{FIELD ESTIMATES OF WEIGHT}

Weight estimates in the field can be made using the same basic techniques utilized for cubic-foot or board-foot volume determinations. An unrealistically simple example will be used to illustrate the procedures, much of which could be computerized. Assume we are interested in total dry weights of species $A$ and $B$, and that those species occur in only two diameter classes, 5 and 10 inches, as follows:

\section{Total dry weight (lbs.)}

\section{$\underline{\text { Dbh }}$}

Species A

100

650
Species B

730 
Table 1

Total tree green weight. ${ }^{*}$

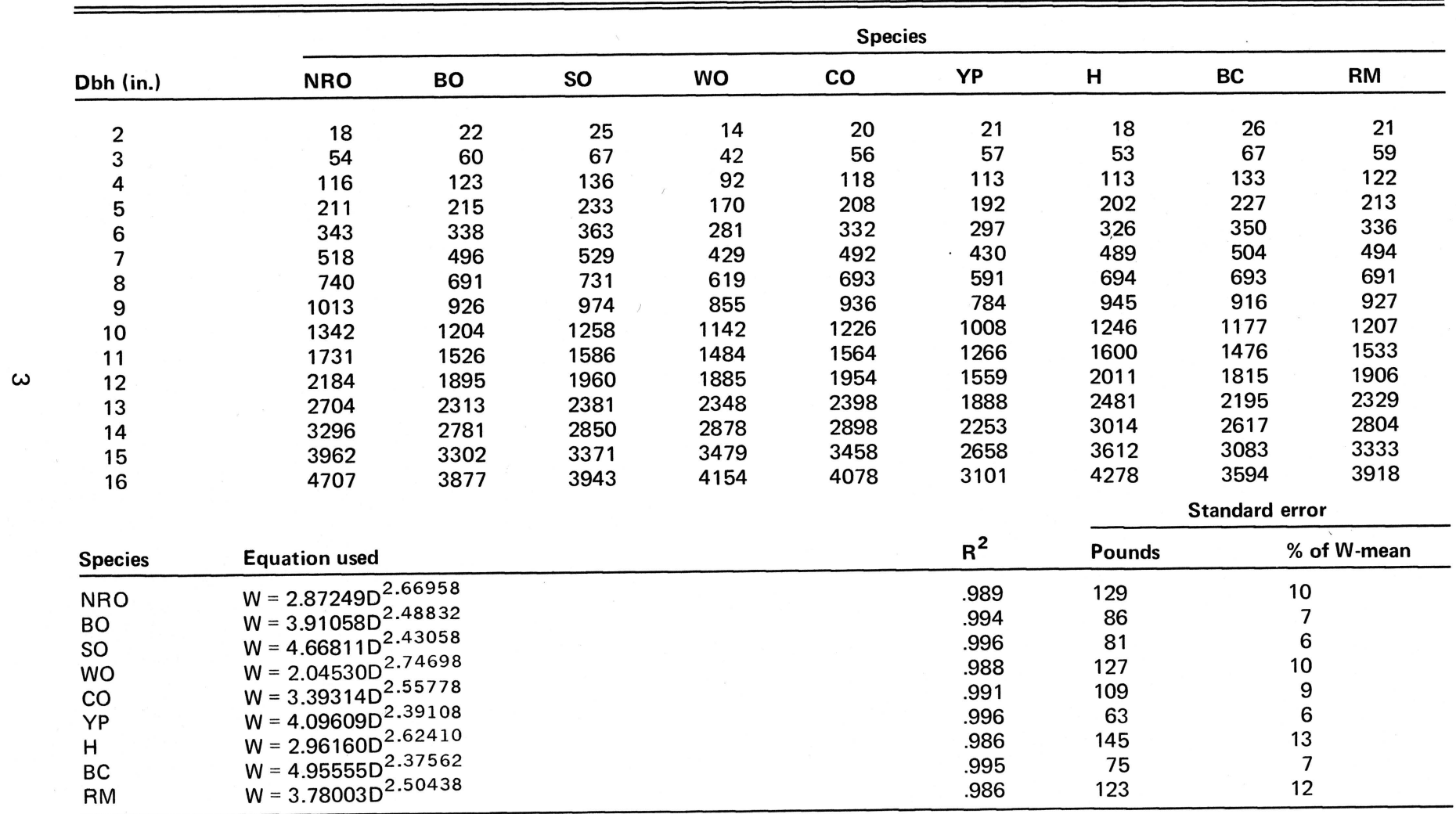

*Weights in tables 1 to 12 are in pounds. 
Table 2

Total tree dry weight.

\begin{tabular}{|c|c|c|c|c|c|c|c|c|c|c|}
\hline \multirow[b]{2}{*}{ Dbh (in.) } & \multicolumn{10}{|c|}{ Species } \\
\hline & NRO & BO & so & wo & $\mathrm{co}$ & YP & $\mathbf{H}$ & BC & & RM \\
\hline 2 & 11 & 12 & 14 & 8 & 12 & 9 & 12 & .14 & & 11 \\
\hline 3 & 31 & 34 & 39 & 25 & 34 & 25 & 34 & 37 & & 30 \\
\hline 4 & 67 & 69 & 78 & 55 & 71 & 52 & 73 & 75 & & 63 \\
\hline 5 & 122 & 121 & 135 & 100 & 125 & 90 & 131 & 128 & & 112 \\
\hline 6 & 198 & 190 & 210 & 163 & 199 & 143 & 212 & 200 & & 179 \\
\hline 7 & 299 & 280 & 306 & 247 & 294 & 211 & 317 & 290 & & 265 \\
\hline 8 & 426 & 391 & 424 & 354 & 412 & 295 & 450 & 401 & & 374 \\
\hline 9 & 583 & 525 & 565 & 487 & 556 & 397 & 613 & 534 & & 505 \\
\hline 10 & 772 & 683 & 731 & 648 & 726 & 517 & 808 & 689 & & 662 \\
\hline 11 & 994 & 867 & 922 & 838 & 924 & 657 & 1037 & 868 & & 845 \\
\hline 12 & 1253 & 1078 & 1141 & 1060 & 1152 & 818 & 1303 & 1072 & & 1056 \\
\hline 13 & 1551 & 1318 & 1386 & 1315 & 1411 & 1000 & 1607 & 1302 & & 1296 \\
\hline 14 & 1888 & 1586 & 1661 & 1607 & 1703 & 1205 & 1951 & 1559 & & 1567 \\
\hline 15 & 2269 & 1885 & 1966 & 1936 & 2028 & 1433 & 2338 & 1842 & & 1870 \\
\hline \multirow[t]{2}{*}{16} & 2694 & 2216 & 2301 & 2305 & 2388 & 1686 & 2769 & 2155 & & 2206 \\
\hline & & & & & & & \multicolumn{4}{|c|}{ Standard error } \\
\hline Species & \multicolumn{5}{|c|}{ Equation used } & $R^{2}$ & Pounds & & \multicolumn{2}{|c|}{$\%$ of $W$-mean } \\
\hline NRO & \multicolumn{5}{|c|}{$W=1.68914 D^{2.65978}$} & .988 & 79 & \multicolumn{3}{|c|}{11} \\
\hline BO & \multicolumn{5}{|c|}{$W=2.14567 D^{2.50304}$} & .994 & 50 & \multicolumn{3}{|c|}{7} \\
\hline so & \multicolumn{5}{|c|}{$W=2.65743 D^{2.43948}$} & .993 & 60 & \multicolumn{3}{|c|}{8} \\
\hline WO & \multicolumn{5}{|c|}{$W=1.28919 D^{2.70096}$} & .988 & 72 & \multicolumn{3}{|c|}{10} \\
\hline $\mathrm{CO}$ & \multicolumn{5}{|c|}{$W=2.12015 D^{2.53442}$} & .991 & 65 & \multicolumn{3}{|c|}{9} \\
\hline YP & \multicolumn{5}{|c|}{$W=1.57792 D^{2.51532}$} & .995 & 37 & \multicolumn{3}{|c|}{7} \\
\hline $\mathrm{H}$ & \multicolumn{5}{|c|}{$W=1.93378 D^{2.62090}$} & .987 & 91 & \multicolumn{3}{|c|}{12} \\
\hline $\mathrm{BC}$ & \multicolumn{5}{|c|}{$W=2.58831 D^{2.42530}$} & .994 & 45 & \multicolumn{3}{|c|}{7} \\
\hline RM & \multicolumn{5}{|c|}{$W=1.81301 D^{2.56226}$} & .981 & 78 & \multicolumn{3}{|c|}{13} \\
\hline
\end{tabular}


Table 3

Total tree dry weight without bark.

\begin{tabular}{|c|c|c|c|c|c|c|c|c|c|c|}
\hline \multirow[b]{2}{*}{ Dbh (in.) } & \multicolumn{10}{|c|}{ Species } \\
\hline & NRO & BO & so & wo & $\mathrm{CO}$ & YP & $\mathrm{H}$ & BC & & RM \\
\hline 2 & 8 & 10 & 11 & 7 & 10 & 8 & 10 & 12 & & 9 \\
\hline 3 & 25 & 27 & 31 & 22 & 28 & 21 & 29 & 33 & & 26 \\
\hline 4 & 55 & 57 & 64 & 48 & 58 & 43 & 62 & 66 & & 55 \\
\hline 5 & 101 & 99 & 112 & 87 & 103 & 76 & 113 & 114 & & 98 \\
\hline 6 & 165 & 157 & 176 & 143 & 164 & 121 & 183 & 178 & & 157 \\
\hline 7 & 250 & 231 & 258 & 216 & 244 & 178 & 276 & 259 & & 233 \\
\hline 8 & 358 & 323 & 360 & 310 & 344 & 249 & 394 & 358 & & 328 \\
\hline 9 & 492 & 434 & 482 & 427 & 465 & 335 & 539 & 478 & & 444 \\
\hline 10 & 655 & 566 & 626 & 567 & 610 & 437 & 713 & 618 & & 583 \\
\hline 11 & 847 & 719 & 794 & 734 & 779 & 555 & 920 & 779 & & 744 \\
\hline 12 & 1071 & 894 & 986 & 929 & 975 & 691 & 1160 & 964 & & 931 \\
\hline 13 & 1330 & 1093 & 1203 & 1153 & 1198 & 846 & 1435 & 1171 & & 1143 \\
\hline 14 & 1624 & 1317 & 1446 & 1409 & 1449 & 1019 & 1749 & 1404 & & 1383 \\
\hline 15 & 1957 & 1566 & 1716 & 1698 & 1731 & 1213 & 2102 & 1661 & & 1651 \\
\hline \multirow[t]{2}{*}{16} & 2330 & 1842 & 2015 & 2022 & 2043 & 1427 & 2496 & 1944 & & 1948 \\
\hline & & & & & & & \multicolumn{4}{|c|}{ Standard error } \\
\hline Species & \multicolumn{5}{|c|}{ Equation used } & $\mathbf{R}^{2}$ & \multicolumn{2}{|l|}{ Pounds } & \multicolumn{2}{|c|}{$\%$ of $W$-mean } \\
\hline & \multirow{2}{*}{\multicolumn{5}{|c|}{$W=1.30152 D^{2.70150}$}} & .988 & 67 & \multicolumn{3}{|c|}{11} \\
\hline BO & & & & $\begin{array}{l}.900 \\
.994\end{array}$ & 42 & \multicolumn{3}{|c|}{7} \\
\hline SO & \multicolumn{5}{|c|}{$W=2.04644 D^{2.48586}$} & .993 & 52 & \multicolumn{3}{|c|}{8} \\
\hline WO & \multicolumn{5}{|c|}{$W=1.12046 D^{2.70442}$} & .987 & 65 & \multicolumn{3}{|c|}{11} \\
\hline $\mathrm{CO}$ & \multicolumn{5}{|c|}{$W=1.63332 D^{2.57224}$} & .989 & 60 & \multicolumn{3}{|c|}{9} \\
\hline YP & \multicolumn{5}{|c|}{$W=1.32004 D^{2.51950}$} & .996 & 29 & \multicolumn{3}{|c|}{6} \\
\hline $\mathrm{H}$ & \multicolumn{5}{|c|}{$W=1.54470 D^{2.66452}$} & .983 & 95 & \multicolumn{3}{|c|}{14} \\
\hline $\mathrm{BC}$ & \multicolumn{5}{|c|}{$W=2.24220 D^{2.44004}$} & .994 & 40 & \multicolumn{3}{|c|}{7} \\
\hline RM & \multicolumn{5}{|c|}{$W=1.57279 D^{2.56866}$} & .982 & 67 & \multicolumn{3}{|c|}{13} \\
\hline
\end{tabular}


Table 4

Total tree dry weight of bark.

\begin{tabular}{|c|c|c|c|c|c|c|c|c|c|c|}
\hline \multirow[b]{2}{*}{ Dbh (in.) } & \multicolumn{10}{|c|}{ Species } \\
\hline & NRO & BO & so & wo & $\mathrm{CO}$ & YP & H & BC & & $\mathrm{RM}$ \\
\hline 2 & 2 & 2 & 3 & 1 & 3 & 2 & 2 & 2 & & 1 \\
\hline 3 & 6 & 6 & 7 & 3 & 7 & 4 & 6 & 4 & & 4 \\
\hline 4 & 12 & 12 & 14 & 7 & 13 & 8 & 11 & 9 & & 8 \\
\hline 5 & 21 & 21 & 22 & 12 & 22 & 15 & 18 & 14 & & 14 \\
\hline 6 & 33 & 33 & 34 & 20 & 34 & 23 & 27 & 22 & & 22 \\
\hline 7 & 48 & 49 & 47 & 30 & 49 & 33 & 39 & 31 & & 32 \\
\hline 8 & 67 & 68 & 63 & 44 & 68 & 46 & 53 & 43 & & 45 \\
\hline 9 & 89 & 91 & 82 & 60 & 89 & 62 & 70 & 56 & & 60 \\
\hline 10 & 116 & 117 & 104 & 79 & 115 & 80 & 89 & 71 & & 79 \\
\hline 11 & 146 & 149 & 128 & 103 & 144 & 101 & 111 & 88 & & 100 \\
\hline 12 & 181 & 184 & 155 & 130 & 177 & 125 & 136 & 108 & & 125 \\
\hline 13 & 221 & 224 & 185 & 161 & 214 & 152 & 164 & 130 & & 152 \\
\hline 14 & 265 & 269 & 218 & 196 & 255 & 182 & 194 & 154 & & 183 \\
\hline 15 & 314 & 319 & 253 & 236 & 300 & 215 & 228 & 181 & & 218 \\
\hline \multirow[t]{2}{*}{16} & 367 & 374 & 292 & 281 & 349 & 252 & 265 & 210 & & 257 \\
\hline & & & & & & & \multicolumn{4}{|c|}{ Standard error } \\
\hline Species & \multicolumn{5}{|c|}{ Equation used } & $\mathbf{R}^{2}$ & Pounds & & \multicolumn{2}{|c|}{$\%$ of $\mathrm{W}$-mean } \\
\hline NRO & \multirow{2}{*}{\multicolumn{5}{|c|}{$W=0.40617 D^{2.45522}$}} & .982 & 14 & & \multicolumn{2}{|l|}{13} \\
\hline $\mathrm{BO}$ & \multirow{2}{*}{\multicolumn{5}{|c|}{$W=0.40547 D^{2.46200}$}} & .993 & 9 & & \multicolumn{2}{|l|}{8} \\
\hline so & \multicolumn{4}{|c|}{$W=0.64595 D^{2.20532}$} & & .991 & 9 & & \multicolumn{2}{|l|}{9} \\
\hline WO & \multicolumn{5}{|c|}{$W=0.16388 D^{2.68560}$} & .985 & 10 & & \multicolumn{2}{|l|}{12} \\
\hline $\mathrm{CO}$ & \multicolumn{5}{|c|}{$W=0.48999 D^{2.36930}$} & .983 & 14 & & \multicolumn{2}{|l|}{12} \\
\hline YP & \multicolumn{5}{|c|}{$W=0.28558 D^{2.44660}$} & .971 & 13 & & \multicolumn{2}{|l|}{16} \\
\hline $\mathrm{H}$ & \multicolumn{5}{|c|}{$W=0.43532 D^{2.31178}$} & .936 & 17 & & \multicolumn{2}{|l|}{22} \\
\hline $\mathrm{BC}$ & \multirow{2}{*}{\multicolumn{5}{|c|}{$W=0.35434 D_{2.30212}^{2.51544}$}} & .987 & 6 & & \multicolumn{2}{|l|}{10} \\
\hline RM & \multicolumn{4}{|c|}{$W=0.24022 D^{2.51544}$} & & .968 & 13 & & \multicolumn{2}{|l|}{18} \\
\hline
\end{tabular}


Table 5

Green weight of branches.

\begin{tabular}{|c|c|c|c|c|c|c|c|c|c|c|}
\hline \multirow[b]{2}{*}{ Dbh (in.) } & \multicolumn{10}{|c|}{ Species } \\
\hline & NRO & BO & so & wo & $\mathrm{CO}$ & YP & $\mathrm{H}$ & $\mathrm{BC}$ & & RM \\
\hline 5 & 107 & 65 & 93 & 70 & 79 & 73 & 79 & 73 & & 93 \\
\hline 6 & 133 & 97 & 107 & 77 & 91 & 52 & 87 & 89 & & 120 \\
\hline 7 & 168 & 135 & 134 & 94 & 113 & 43 & 112 & 110 & & 151 \\
\hline 8 & 212 & 181 & 173 & 122 & 148 & 46 & 153 & 135 & & 186 \\
\hline 9 & 266 & 234 & 225 & 160 & 193 & 61 & 209 & 165 & & 224 \\
\hline 10 & 328 & 294 & 289 & 208 & 249 & 87 & 281 & 199 & & 267 \\
\hline 11 & 400 & 362 & 366 & 266 & 317 & 126 & 368 & 238 & & 314 \\
\hline 12 & 481 & 437 & 456 & 335 & 396 & 176 & 472 & 282 & & 365 \\
\hline 13 & 571 & 521 & 558 & 414 & 487 & 237 & 591 & 330 & & 419 \\
\hline 14 & 670 & 612 & 673 & 503 & 588 & 311 & 726 & 382 & & 478 \\
\hline 15 & 779 & 711 & 800 & 603 & 701 & 396 & 877 & 439 & & 541 \\
\hline \multirow[t]{2}{*}{16} & 896 & 818 & 940 & 712 & 825 & 493 & 1043 & 501 & & 608 \\
\hline & & & & & & & \multicolumn{4}{|c|}{ Standard error } \\
\hline Species & \multicolumn{5}{|l|}{ Equation used } & $\mathrm{R}^{2}$ & \multicolumn{2}{|l|}{ Pounds } & \multicolumn{2}{|c|}{$\%$ of $\mathrm{W}$-mean } \\
\hline NRO & \multirow{2}{*}{\multicolumn{5}{|c|}{$\begin{array}{l}W=116.07240+4.58936 D^{2}-24.66151 D \\
W=195802 D^{2.17664}\end{array}$}} & .848 & 109 & & \multicolumn{2}{|l|}{30} \\
\hline $\mathrm{BO}$ & & & \multicolumn{3}{|c|}{$W=1.95802 D^{2.17664}$} & .892 & 84 & & \multicolumn{2}{|l|}{24} \\
\hline so & \multicolumn{5}{|c|}{$W=212.75897+6.30761 D^{2}-55.44747 D$} & .952 & 65 & & \multicolumn{2}{|l|}{18} \\
\hline WO & \multicolumn{5}{|c|}{$W=190.10052+5.14752 D^{2}-49.71524 D$} & .959 & 44 & & \multicolumn{2}{|l|}{16} \\
\hline $\mathrm{CO}$ & \multicolumn{5}{|c|}{$W=189.61831+5.62049 D^{2}-50.21763 D$} & .955 & 52 & & \multicolumn{2}{|l|}{17} \\
\hline YP & \multicolumn{5}{|c|}{$W=352.07486+5.87535 D^{2}-85.21641 D$} & .839 & 61 & & \multicolumn{2}{|l|}{39} \\
\hline $\mathrm{H}$ & \multicolumn{5}{|c|}{$W=270.38361+7.87893 D^{2}-77.76552 D$} & .838 & 120 & & \multicolumn{2}{|l|}{38} \\
\hline $\mathrm{BC}$ & \multicolumn{5}{|c|}{$W=59.17315+2.26508 D^{2}-8.62607 D$} & .820 & 62 & & \multicolumn{2}{|l|}{29} \\
\hline RM & \multicolumn{5}{|c|}{$W=20.05467+2.00411 D^{2}+4.66393 D$} & .827 & 68 & & \multicolumn{2}{|l|}{26} \\
\hline
\end{tabular}


Table 6

Dry weight of branches.

\begin{tabular}{|c|c|c|c|c|c|c|c|c|c|c|}
\hline \multirow[b]{2}{*}{ Dbh (in.) } & \multicolumn{10}{|c|}{ Species } \\
\hline & NRO & BO & so & Wo & CO & YP & $\mathbf{H}$ & BC & & $\mathbf{R M}$ \\
\hline 5 & 62 & 48 & 58 & 46 & 45 & 33 & 50 & 39 & & 49 \\
\hline 6 & 78 & 58 & 64 & 45 & 51 & 26 & 54 & 49 & & 64 \\
\hline 7 & 99 & 75 & 78 & 51 & 65 & 23 & 69 & 62 & & 82 \\
\hline 8 & 126 & 99 & 101 & 64 & 85 & 25 & 95 & 76 & & 101 \\
\hline 9 & 158 & 129 & 132 & 83 & 111 & 33 & 131 & 93 & & 122 \\
\hline 10 & 196 & 166 & 172 & 110 & 145 & 45 & 178 & 112 & & 145 \\
\hline 11 & 239 & 211 & 220 & 143 & 185 & 62 & 236 & 133 & & 170 \\
\hline 12 & 287 & 262 & 276 & 184 & 232 & 85 & 304 & 156 & & 197 \\
\hline 13 & 342 & 320 & 340 & 231 & 285 & 112 & 383 & 181 & & 225 \\
\hline \multirow[t]{4}{*}{$\infty$} & 401 & 385 & 413 & 285 & 345 & 144 & 473 & 209 & & 256 \\
\hline & 466 & 456 & 494 & 346 & 412 & 182 & 574 & 238 & & 288 \\
\hline & 537 & 535 & 584 & 413 & 486 & 224 & 685 & 270 & & 323 \\
\hline & & & & & & & \multicolumn{4}{|c|}{ Standard error } \\
\hline Species & \multicolumn{5}{|l|}{ Equation used } & $\mathbf{R}^{2}$ & \multicolumn{2}{|l|}{ Pounds } & \multicolumn{2}{|c|}{$\%$ of $W$-mean } \\
\hline NRO & \multicolumn{5}{|c|}{$W=66.63842+2.75334 D^{2}-14.64401 D$} & .845 & 66 & \multicolumn{3}{|c|}{30} \\
\hline $\mathrm{BO}$ & \multicolumn{5}{|c|}{$W=101.75593+3.43396 D^{2}-27.87506 D$} & .888 & 54 & \multicolumn{3}{|c|}{26} \\
\hline so & \multicolumn{5}{|c|}{$W=152.09258+4.16948 D^{2}-39.72114 D$} & .943 & 44 & \multicolumn{3}{|c|}{20} \\
\hline WO & \multicolumn{5}{|c|}{$W=153.17470+3.42784 D_{2}^{2}-38.59326 D$} & .902 & 41 & \multicolumn{3}{|c|}{26} \\
\hline $\mathrm{CO}$ & \multicolumn{5}{|c|}{$W=112.26122+3.35130 D^{2}-30.27026 D$} & .961 & 29 & \multicolumn{3}{|c|}{16} \\
\hline YP & \multicolumn{5}{|c|}{$W=146.29742+2.49589 D_{0}^{2}-35.08911 D$} & .732 & 38 & \multicolumn{3}{|c|}{51} \\
\hline $\mathrm{H}$ & \multicolumn{5}{|c|}{$W=191.16897+5.36555 D^{2}-54.98699 D$} & .842 & 78 & \multicolumn{3}{|c|}{38} \\
\hline $\mathrm{BC}$ & \multicolumn{5}{|c|}{$W=20.48931+1.07958 D^{2}-1.67602 D$} & .811 & 35 & \multicolumn{3}{|c|}{29} \\
\hline RM & \multicolumn{5}{|c|}{$W=0.93846 D^{2}+5.18726 D-0.55683$} & .793 & 41 & \multicolumn{3}{|c|}{29} \\
\hline
\end{tabular}


Table 7

Dry weight of branches without bark.




Table 8

Dry weight of branch bark.

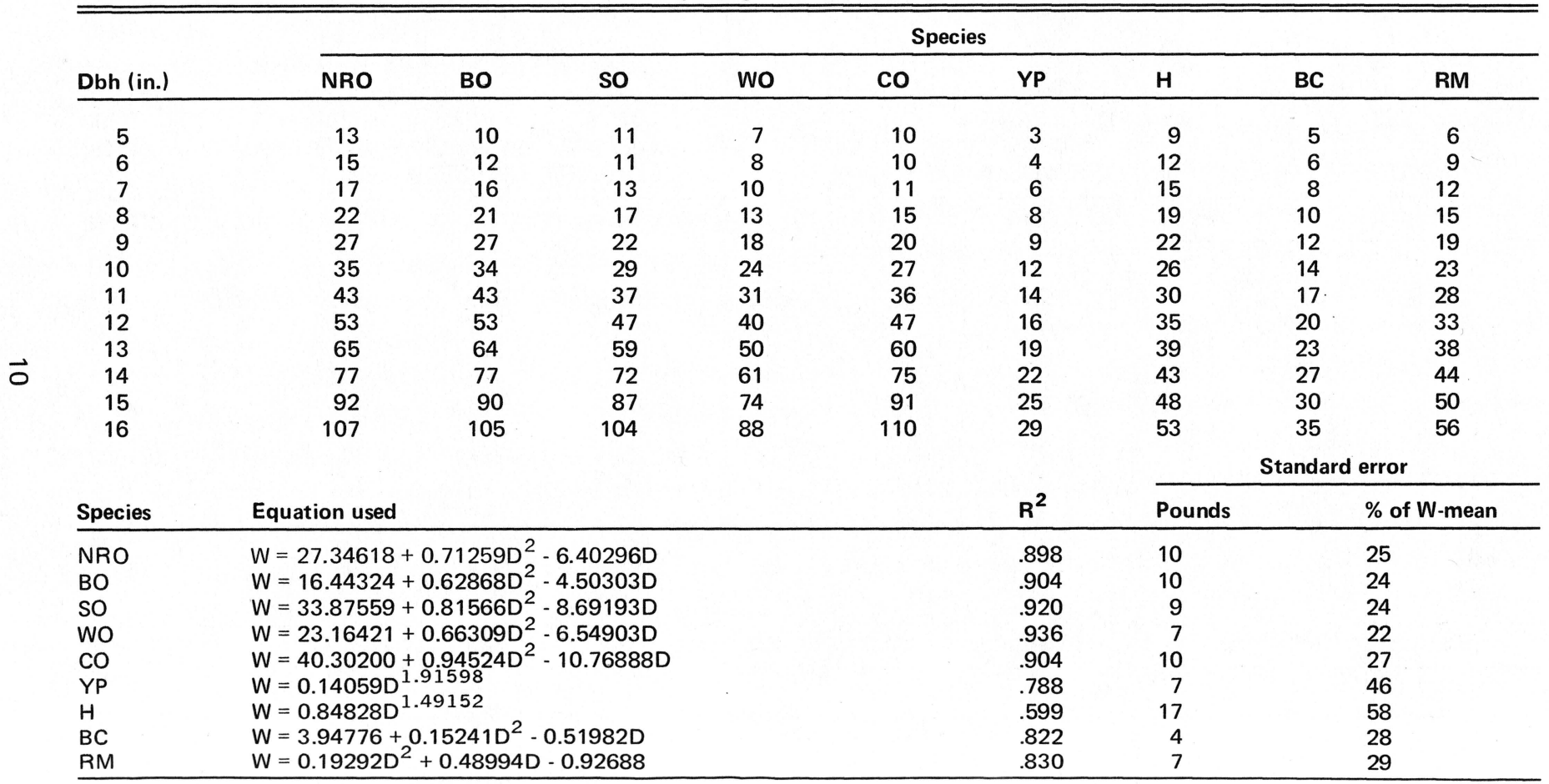


Table 9

Green weight to a 4-inch dob.

\begin{tabular}{|c|c|c|c|c|c|c|c|c|c|c|}
\hline \multirow[b]{2}{*}{ Dbh (in.) } & \multicolumn{10}{|c|}{ Species } \\
\hline & NRO & BO & so & wo & co & YP & H & BC & & $\mathbf{R M}$ \\
\hline 5 & 162 & 222 & 184 & 116 & 144 & 121 & 92 & 165 & & 203 \\
\hline 6 & 273 & 280 & 286 & 236 & 258 & 221 & 168 & 264 & & 239 \\
\hline 7 & 415 & 375 & 417 & 384 & 400 & 349 & 280 & 396 & & 326 \\
\hline 8 & 589 & 507 & 578 & 560 & 571 & 505 & 435 & 561 & & 464 \\
\hline 9 & 794 & 677 & 767 & 765 & 769 & 690 & 641 & 758 & & 653 \\
\hline 10 & 1032 & 885 & 985 & 998 & 996 & 904 & 907 & 988 & & 893 \\
\hline 11 & 1301 & 1130 & 1232 & 1259 & 1252 & 1146 & 1241 & 1251 & & 1185 \\
\hline 12 & 1602 & 1413 & 1508 & 1549 & 1535 & 1416 & 1653 & 1547 & & 1528 \\
\hline 13 & 1935 & 1733 & 1812 & 1866 & 1846 & 1716 & 2152 & 1875 & & 1922 \\
\hline 14 & 2300 & 2091 & 2146 & 2213 & 2186 & 2044 & 2747 & 2236 & & 2368 \\
\hline 15 & 2696 & 2486 & 2509 & 2587 & 2554 & 2400 & 3448 & 2630 & & 2864 \\
\hline \multirow[t]{2}{*}{16} & 3124 & 2919 & 2901 & 2990 & 2950 & 2785 & 4226 & 3057 & & 3412 \\
\hline & & & & & & & \multicolumn{4}{|c|}{ Standard error } \\
\hline Species & \multicolumn{5}{|c|}{ Equation used } & $\mathbf{R}^{2}$ & Pounds & \multicolumn{3}{|c|}{$\%$ of $\mathrm{W}$-mean } \\
\hline NRO & \multicolumn{5}{|c|}{$W=88.21958+15.90187 D^{2}-64.67165 D$} & .988 & 108 & \multicolumn{3}{|c|}{9} \\
\hline $\mathrm{BO}$ & \multicolumn{5}{|c|}{$W=497.81493+18.76768 D^{2}-148.94424 D$} & .985 & 104 & \multicolumn{3}{|c|}{9} \\
\hline SO & \multicolumn{5}{|c|}{$W=108.22596+14.48597 D^{2}-57.22531 D$} & .979 & 136 & \multicolumn{3}{|c|}{12} \\
\hline WO & \multicolumn{5}{|c|}{$W=14.14272 D^{2}-35.74697 D-58.95854$} & .983 & 127 & \multicolumn{3}{|c|}{12} \\
\hline $\mathrm{CO}$ & \multicolumn{5}{|c|}{$W=14.10156 D^{2}-41.01997 D-3.56707$} & .992 & 82 & \multicolumn{3}{|c|}{8} \\
\hline YP & \multicolumn{5}{|c|}{$W=52.83929+14.27865 D^{2}-57.70586 D$} & .971 & 151 & \multicolumn{3}{|c|}{14} \\
\hline $\mathrm{H}$ & \multicolumn{5}{|c|}{$W=0.45983 D^{3.29484}$} & .969 & 171 & \multicolumn{3}{|c|}{17} \\
\hline $\mathrm{BC}$ & \multicolumn{5}{|c|}{$W=162.72473+16.39759 D^{2}-81.44786 D$} & .987 & 105 & \multicolumn{3}{|c|}{9} \\
\hline $\mathrm{RM}$ & \multicolumn{5}{|c|}{$W=794.01142+25.61191 D^{2}-246.17225 D$} & .979 & 132 & \multicolumn{3}{|c|}{13} \\
\hline
\end{tabular}


Table 10

Dry weight to a 4-inch dob.

\begin{tabular}{|c|c|c|c|c|c|c|c|c|c|c|}
\hline \multirow[b]{2}{*}{ Dbh (in.) } & \multicolumn{10}{|c|}{ Species } \\
\hline & NRO & BO & so & wo & $\mathrm{CO}$ & YP & $\mathrm{H}$ & $\mathrm{BC}$ & & RM \\
\hline 5 & 91 & 124 & 88 & 85 & 99 & 64 & 61 & 93 & & 105 \\
\hline 6 & 156 & 156 & 142 & 138 & 157 & 114 & 110 & 161 & & 129 \\
\hline 7 & 238 & 209 & 212 & 207 & 232 & 180 & 183 & 245 & & 180 \\
\hline 8 & 338 & 284 & 301 & 294 & 325 & 261 & 284 & 346 & & 259 \\
\hline 9 & 455 & 380 & 409 & 400 & 438 & 358 & 417 & 462 & & 366 \\
\hline 10 & 590 & 499 & 539 & 528 & 571 & 471 & 590 & 595 & & 500 \\
\hline 11 & 743 & 639 & 691 & 678 & 726 & 600 & 806 & 744 & & 662 \\
\hline 12 & 913 & 800 & 868 & 852 & 904 & 745 & 1073 & 909 & & 851 \\
\hline 13 & 1101 & 984 & 1070 & 1052 & 1107 & 905 & 1395 & 1091 & & 1069 \\
\hline 14 & 1307 & 1189 & 1299 & 1278 & 1334 & 1082 & 1779 & 1288 & & 1314 \\
\hline 15 & 1530 & 1416 & 1556 & 1532 & 1588 & 1274 & 2231 & 1502 & & 1586 \\
\hline \multirow[t]{2}{*}{16} & 1771 & 1665 & 1842 & 1815 & 1869 & 1482 & 2758 & 1732 & & 1887 \\
\hline & & & & & & & \multicolumn{4}{|c|}{ Standard error } \\
\hline Species & \multicolumn{5}{|l|}{ Equation used } & $\mathbf{R}^{2}$ & Pounds & & \multicolumn{2}{|c|}{$\%$ of $\mathrm{W}$-mean } \\
\hline NRO & & .984 & 70 & \multicolumn{3}{|c|}{11} \\
\hline $\mathrm{BO}$ & \multicolumn{5}{|c|}{$W=293.09646+10.86577 D^{2}-88.11460 D$} & .987 & 55 & \multicolumn{3}{|c|}{9} \\
\hline SO & \multicolumn{5}{|c|}{$W=1.30430 D^{2.61602}$} & .979 & 76 & \multicolumn{3}{|c|}{11} \\
\hline WO & \multicolumn{5}{|c|}{$W=1.24354 D^{2.62792}$} & .989 & 55 & \multicolumn{3}{|c|}{8} \\
\hline $\mathrm{CO}$ & \multicolumn{5}{|c|}{$W=1.71389 D^{2.52260}$} & .983 & 69 & \multicolumn{3}{|c|}{10} \\
\hline YP & \multicolumn{5}{|c|}{$W=53.24629+7.91561 D^{2}-37.34973 D$} & .964 & 90 & \multicolumn{3}{|c|}{16} \\
\hline $\mathrm{H}$ & \multicolumn{5}{|c|}{$W=0.30832 D^{3.28170}$} & .972 & 106 & \multicolumn{3}{|c|}{16} \\
\hline $\mathrm{BC}$ & \multicolumn{5}{|c|}{$W=8.09971 D^{2}-21.14376 D-3.40207$} & .982 & 70 & \multicolumn{3}{|c|}{11} \\
\hline $\mathrm{RM}$ & \multicolumn{5}{|c|}{$W=402.37775+13.83454 D^{2}-128.58822 D$} & .976 & 78 & \multicolumn{3}{|c|}{14} \\
\hline
\end{tabular}


Table 11

Dry weight without bark to a 4-inch dob.

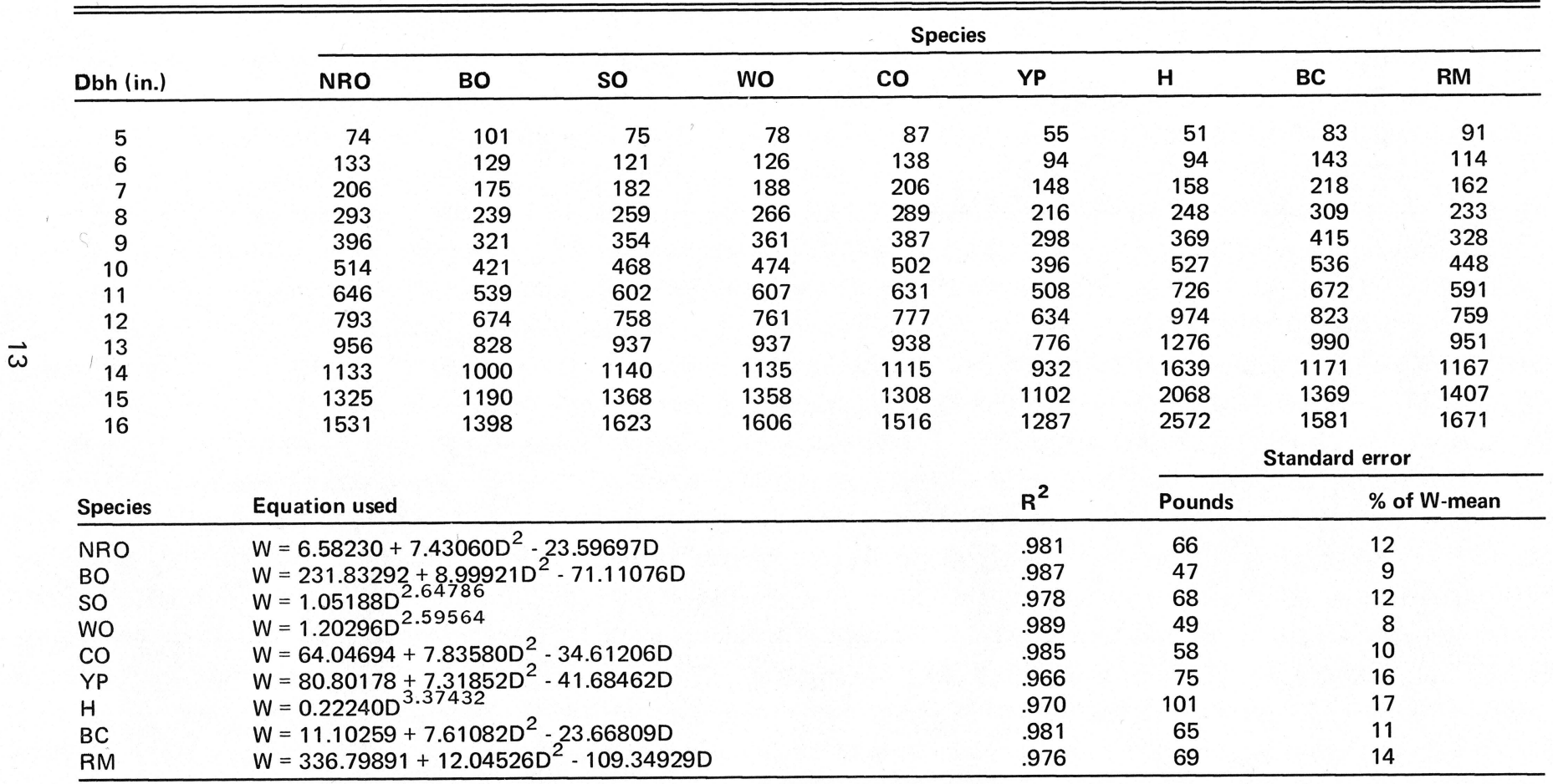


Table 12

Dry weight of bark to a 4-inch dob.

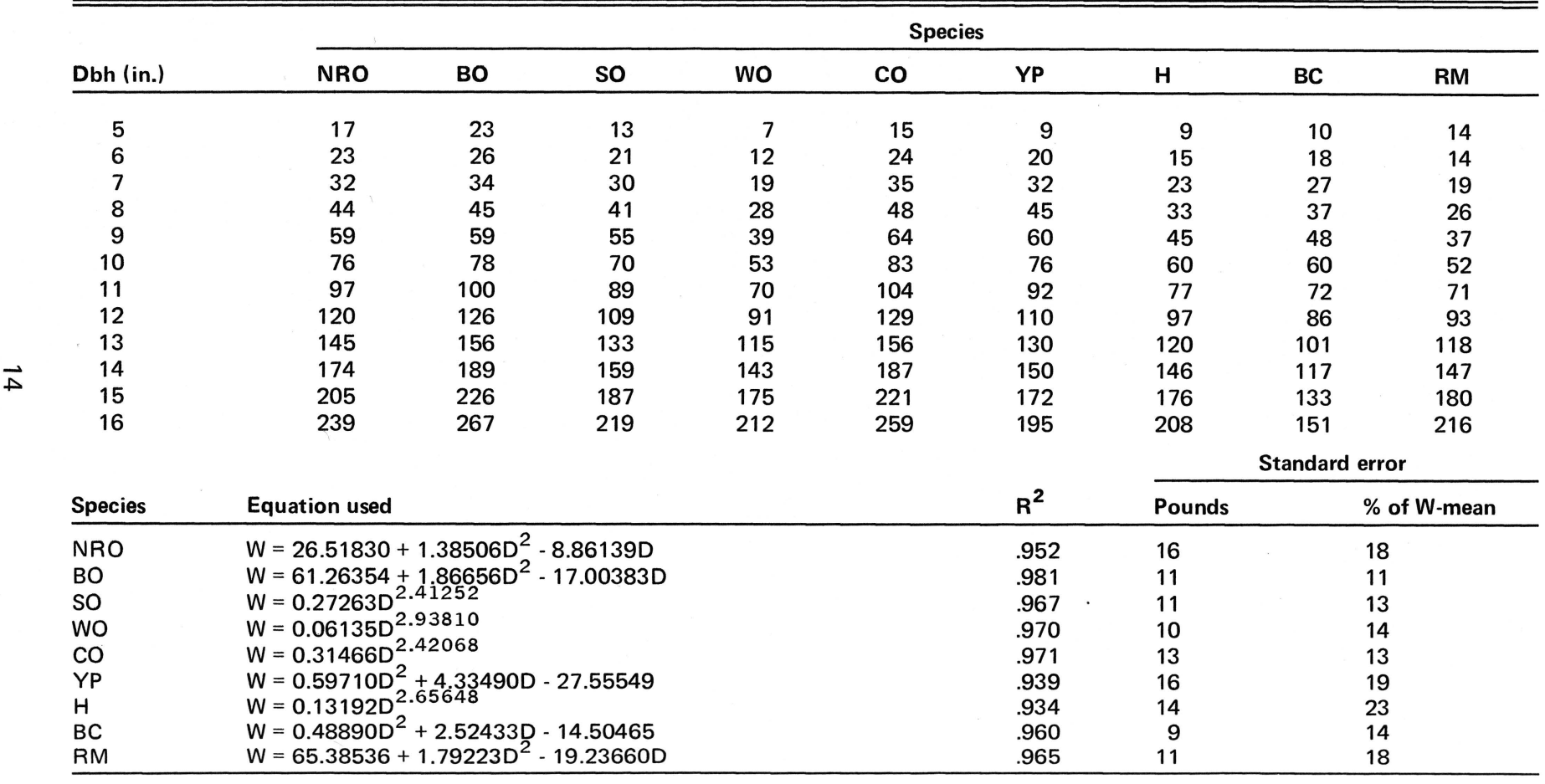




\section{Weight Estimates When Marking Timber}

Weight estimates are easily derived when each tree to be harvested is visited and marked with paint. A tally of the number of trees by species and diameter is made. For example:

Number of trees

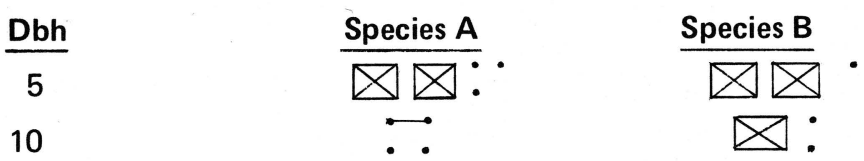

Weight estimates are:

\begin{tabular}{|c|c|c|c|c|c|}
\hline Dbh & Species A & & Species B & & Totals \\
\hline 5 & $(23)(100)=$ & $=2300$ & $(21)(125)$ & $=2625$ & 4925 \\
\hline 10 & (5) $(650)=$ & 3250 & $(12)(730)$ & $=8760$ & 12010 \\
\hline Totals & & 5550 & & 11385 & 16935 \\
\hline
\end{tabular}

Our total weight estimate is 16935 pounds, and, as each member of the population of interest has been measured, there is no sampling error in the statistical sense.

\section{Weight Estimates Using Fixed-Area Plots}

If cruising is done using fixed-area plots, a separate tally of trees by species and diameter class is made at each plot. Suppose the following data are collected on $1 / 10$-acre plots:

\begin{tabular}{|c|c|c|c|}
\hline \multirow[b]{2}{*}{ Plot } & \multirow[b]{2}{*}{ Dbh } & \multicolumn{2}{|c|}{ Number of trees } \\
\hline & & Species A & Species B \\
\hline \multirow[t]{2}{*}{1} & 5 & $\because$ & $\cdot \bullet$ \\
\hline & 10 & - & - \\
\hline \multirow[t]{2}{*}{2} & 5 & & \\
\hline & 10 & & \\
\hline \multirow[t]{2}{*}{3} & 5 & - & \\
\hline & 10 & & .. \\
\hline \multirow[t]{2}{*}{4} & 5 & & \\
\hline & 10 & $\cdot \cdot$ & \\
\hline
\end{tabular}


Weight estimates for plots are:

\begin{tabular}{|c|c|c|c|c|c|}
\hline Plot & Dbh & Species A & Species B & Totals & \\
\hline \multirow[t]{2}{*}{1} & 5 & $(6)(100)=600$ & $(2)(125)=250$ & 850 & \multirow{2}{*}{2230} \\
\hline & 10 & (1) $(650)=650$ & $(1)(730)=730$ & 1380 & \\
\hline 2 & & - & - & 0 & 0 \\
\hline \multirow[t]{2}{*}{3} & 5 & $(1)(100)=100$ & - & 100 & \multirow{2}{*}{3020} \\
\hline & 10 & - & (4) $(730)=2920$ & 2920 & \\
\hline \multirow[t]{2}{*}{4} & 5 & $(1)(100)=100$ & $(1)(125)=125$ & $225]$ & \multirow{2}{*}{1525} \\
\hline & 10 & (2) $(650)=1300$ & - & 1300 & \\
\hline & & 2750 & 4025 & 6775 & \\
\hline
\end{tabular}

Average per-acre estimates are:

Dbh

5

10
Species A

(10) $\left[\frac{600+100+100}{4}\right]=2000$

(10) $\left[\frac{650+1300}{4}\right]=4875$

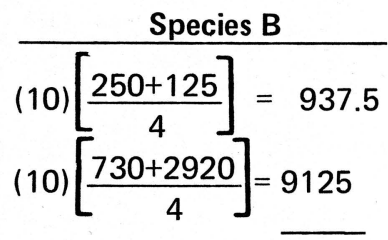

$10062.5 \quad 16937.5$

Total

2937.5

14000

Totals

6875

The average $1 / 10$-acre estimate is $6775 / 4=1694$ pounds. The standard error $\left(\mathrm{S}_{\overline{\mathrm{x}}}\right)$ for that estimate is:

$$
S_{\bar{x}}=\sqrt{\frac{\Sigma X^{2}-(\Sigma X)^{2} / n}{n(n-1)}}
$$

where: $X=$ weight estimate for a given plot

$\mathrm{n}=$ number of plots

Therefore:

$$
S_{\bar{x}}=\sqrt{\frac{(2230)^{2}+(0)^{2}+(3020)^{2}+(1525)^{2}-(6775)^{2} / 4}{4(4-1)}}=642
$$

Expressed as a percent of the mean, our sampling error is:

$$
\left[\frac{642}{1694}\right](100)=38 \%
$$


On an acre basis, our weight estimate is:

$$
\text { (10) } \begin{gathered}
(1694 \pm 642)=16940 \pm 6420 \text { pounds } \\
\text { or } 16940 \pm 38 \%
\end{gathered}
$$

About two in three times we expect the true population mean to be within these limits. Limits are doubled for a 19 in 20 chance or tripled for a 99 in 100 chance. The usual assumptions of random sampling are made here, of course.

\section{Weight Estimates Using Point Samples}

If point sampling $(B A F=10)$ is to be used, weights in the basic table are multiplied by the appropriate conversion factors (see Kulow 1965), as follows:

\begin{tabular}{|c|c|c|}
\hline Dbh & Species A & Species B \\
\hline 5 & $(73.34)(100)=7334$ & $(73.34)(125)=9168$ \\
\hline 10 & $(18.34)(650)=11921$ & $(18.34)(730)=13388$ \\
\hline
\end{tabular}

\section{Total dry weight (Ibs.) per acre per in-tree ${ }^{2}$}

\begin{tabular}{|c|c|c|c|}
\hline \multirow[b]{2}{*}{ Point } & \multirow[b]{2}{*}{ Dbh } & \multicolumn{2}{|c|}{ Number of in-trees } \\
\hline & & Species A & Species B \\
\hline \multirow[t]{2}{*}{1} & 5 & $\cdot \cdot$ & \\
\hline & 10 & • & • \\
\hline \multirow[t]{2}{*}{2} & 5 & & \\
\hline & 10 & & \\
\hline \multirow[t]{2}{*}{3} & 5 & & \\
\hline & 10 & & $\dot{0}$ \\
\hline \multirow[t]{2}{*}{4} & 5 & $\stackrel{0}{\bullet}$ & \\
\hline & 10 & & \\
\hline
\end{tabular}

The following data are collected:

${ }^{2}$ An "in-tree" is a tree selected as "in" with a prism or similar instrument. 
Weight estimates per acre for points are:

\begin{tabular}{|c|c|c|c|c|c|c|c|}
\hline Point & Dbh & Species & A & Species & B & Totals & \\
\hline 1 & 5 & $(2)(7334)=$ & 14668 & $(1)(9168)=$ & 9168 & 23836 & \\
\hline & 10 & $(1)(11921)=$ & $=11921$ & (1) $(13388)=$ & 13388 & 25309 & \\
\hline 2 & & - & & - & & & \\
\hline 3 & 5 & - & & - & & & \\
\hline & 10 & - & & (4) $(13388)=$ & 53552 & 53552 & \\
\hline 4 & 5 & (5) $(7334)=$ & 36670 & - & & 36670 & \\
\hline & 10 & - & & - & & & \\
\hline 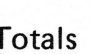 & & & 63259 & & 76108 & & 139367 \\
\hline
\end{tabular}

Average per-acre estimates are:

$\begin{array}{lll}\frac{\text { Dbh }}{5} & \frac{\text { Species A }}{\left[\frac{14668+36670}{4}\right]=12835} \frac{\text { Species B }}{\left[\frac{9168}{4}\right]=} \frac{2292}{15127} \\ 10 & {\left[\frac{11921]}{4}\right]=\frac{2980}{15815}\left[\frac{13388+53552}{4}\right]=1673519715}\end{array}$

Totals

The average per-acre estimate is $\mathbf{3 4 8 4 2}$ pounds. The standard error is:

$$
\begin{aligned}
S_{\bar{x}} & =\sqrt{\frac{(49145)^{2}+(0)^{2}+(53552)^{2}+(36670)^{2}-(139367)^{2} / 4}{4(4-1)}} \\
& =12152
\end{aligned}
$$

Sampling error, as a percent, is:

$$
\left[\frac{12152}{34842}\right](100)=35 \%
$$

\section{Weight Estimates Using Point and 3P Sampling}

Much time can be saved in the field by combining $3 \mathrm{P}$ and point sampling. Again, we will assume $B A F=10$. Before the cruise, determine: 
(1) the number of point samples desired. This can be done statistically (see Wiant 1976), but in most situations will be at least 30 and not more than 100.

(2) the number of 3P sample points needed for the desired accuracy. Wiant (1976) provides a formula for a statistical determination, but 15 to 20 should suffice in most cases.

(3) the approximate sum of basal areas ( $\approx \mathrm{KPI})$ expected at the point samples. For example, if you assume point samples will average 40 square feet of basal area, and you plan to have 100 point samples:

$$
z \mathrm{KPI}=(40)(100)=4000
$$

Develop a list of random numbers, one for each point sample, from 1 through $K Z$, where:

$$
K Z=\frac{\approx K P I}{\text { number of } 3 P \text { samples desired }}
$$

If 20,3P samples are desired:

$$
\begin{aligned}
K Z & =\frac{4000}{20} \\
& =200
\end{aligned}
$$

In the field, record the number of in-trees by species on a point sample. Then, total the number of in-trees and multiply by 10 to obtain the per-acre basal area estimate for that point sample.

If that basal area is less than the random number for that point sample, go the next point. If that basal area equals or exceeds the random number for that point, measure the dbh of each in-tree.

After field work is completed, calculate:

(1) the ratio of per-acre weight $(Y)$ at each $3 P$ point sample to the basal area at that point sample, or $\mathrm{Y} / \mathrm{KPI}$.

(2) the total per-acre volume estimate, which equals the average basal area on all point samples times the average $\mathrm{Y} / \mathrm{KPI}$-ratio. That ratio times the average basal area for a given species provides a per-acre weight estimate for that species; however, if there are sufficient data, an average $\mathrm{Y} / \mathrm{KPI}$-ratio should be calculated for the individual species to provide a better estimate.

(3) the approximate sampling error, which includes that due to the point samples and that related to $3 \mathrm{P}$, plus a covariance term which will be ignored in this paper as is usually done in practice. 
As an illustration of calculations, the example in the previous section will be used:

\begin{tabular}{|c|c|c|c|c|c|c|}
\hline \multirow[b]{2}{*}{ Point } & \multirow[b]{2}{*}{ Random no. } & \multicolumn{3}{|c|}{$\Sigma \mathrm{KPI}$} & \multirow[b]{2}{*}{$Y$} & \multirow[b]{2}{*}{$\mathrm{Y} / \mathrm{KPI}$} \\
\hline & & Species A & Species B & Total & & \\
\hline 1 & 23 & 30 & 20 & 50 & 49145 & 983 \\
\hline 2 & 11 & 0 & 0 & 0 & - & - \\
\hline 3 & 8 & 0 & 40 & 40 & 53552 & 1339 \\
\hline 4 & 64 & 50 & 0 & 50 & - & - \\
\hline & tal & 80 & 60 & 140 & & 2322 \\
\hline & verage & 20 & 15 & 35 & & 1161 \\
\hline
\end{tabular}

Total per-acre weight $=(35)(1161)=40635$

Species A per-acre weight $=(20)(1161)=23220$

If there were sufficient data, a better estimate for Species $A$ is obtained by calculating its own average $\mathrm{Y} / \mathrm{KPI}$-ratio, as:

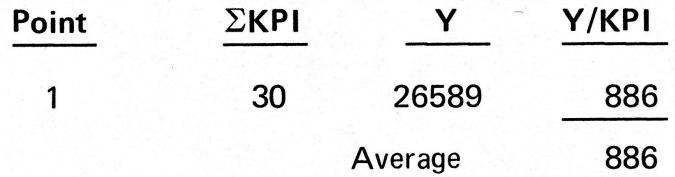

Per-acre weight $=(20)(886)=17720$

Species B per-acre weight $=(15)(1161)=17415$

Again, a better estimate for species $B$ is:

\begin{tabular}{|c|c|c|c|}
\hline Point & $\Sigma K P I$ & $Y$ & $\mathrm{Y} / \mathrm{KPI}$ \\
\hline 1 & 20 & 22556 & 1128 \\
\hline \multirow[t]{2}{*}{3} & 40 & 53552 & 1339 \\
\hline & & erage & 1234 \\
\hline
\end{tabular}

Per-acre weight $=(15)(1234)=18510$

When separate $\mathrm{Y} / \mathrm{KPI}$-ratios are calculated for the different species for weight estimates, the sums of per-acre weight estimates for all species may not equal the total per-acre estimate, but the difference should be minor.

The standard error for point samples is based on basal area values:

$$
\begin{aligned}
S_{\bar{x}}^{-} & =\sqrt{\frac{(50)^{2}+(0)^{2}+(40)^{2}+(50)^{2}-(140)^{2} / 4}{4(4-1)}} \\
& =11.9
\end{aligned}
$$


The standard error for $3 \mathrm{P}$ samples is based on the $\mathrm{Y} / \mathrm{KPI}$-ratios:

$$
\begin{aligned}
S_{\bar{x}} & =\sqrt{\frac{(983)^{2}+(1339)^{2}-(2322)^{2} / 2}{2(2-1)}} \\
& =178
\end{aligned}
$$

The sampling error as a percent for the cruise

$=(100)$

$$
\sqrt{\left[\frac{\mathrm{S}_{\mathrm{x}}^{-} \text {for point samples }}{\text { average basal area }}\right]^{2}+\left[\frac{\mathrm{S}_{\mathrm{x}}^{-} \text {for } \mathrm{Y} / \mathrm{KPI} \text {-ratios }}{\text { average } \mathrm{Y} / \mathrm{KPI} \text {-ratio }}\right]^{2}}
$$

$=(100)$

$$
\sqrt{\left[\frac{11.9}{35}\right]^{2}+\left[\frac{178}{1161}\right]^{2}}
$$

$=37 \%$

The per-acre weight estimate is, then, $40635 \pm 37 \%$.

Tables 13 to 24 are provided for point sample estimates using these techniques $(\mathrm{BAF}=10)$.

\section{Computerized Approach}

As Lenhart et al. (1973) point out, formulae, such as those in tables 1 to 12 , can be modified for computerized applications. Using the one for total tree green weight for northern red oak as an example:

$$
W=2.87249 D^{2.66958}
$$

which is divided by $0.00545415 D^{2}$, the basal area (BA) in square feet for a diameter class, expressed in inches:

$$
\frac{W}{0.00545415 D^{2}}=\frac{2.87249 D^{2.66958}}{0.00545415 D^{2}}
$$

giving:

$$
\mathrm{W} / \mathrm{BA}=526.66135 \mathrm{D}^{0.66958}
$$

Suppose a single point sample tally $(B A F=10)$ of northern red oak is as follows:

$\begin{array}{cl}\frac{\text { Dbh }}{5} & \text { No. in-trees } \\ 6 & : \\ 7 & : \\ 7 & -\end{array}$


Using Table 13, the per-acre total tree green weight estimate is:

\begin{tabular}{|c|c|}
\hline Dbh & Per-acre weight \\
\hline 5 & $(2)(15472)=30944$ \\
\hline 6 & (3) $(17481)=52443$ \\
\hline 7 & $(1)(19382)=19382$ \\
\hline & 102769 \\
\hline
\end{tabular}

Using the formula approach, W/BA is calculated for each tree, summed, and multiplied by the BAF, as follows:

$$
\begin{aligned}
\text { W/BA } & =\left[526.66135(5)^{0.66958}\right](2)=3094.4 \\
& =\left[526.66135(6)^{0.66958}\right](3)=5244.3 \\
& =\left[526.66135(7)^{0.66958}\right](1)=\frac{1938.2}{10276.9}
\end{aligned}
$$

$(B A F=10)(10276.9)=102769$ pounds per acre, the same answer obtained using Table 13.

In actual practice, in-trees should be measured to the nearest tenth inch.

Equations of the form exemplified by the green weight of branches for northern red oak are modified as follows:

$$
\begin{aligned}
W & =116.07240+4.58936 D^{2}-24.66151 D \\
W / B A & =\frac{116.07240}{0.00545415 D^{2}}+\frac{4.58936 D^{2}}{0.00545415 D^{2}}-\frac{24.66151 D}{0.00545415 D^{2}} \\
& =21281.48291 D^{-2}+841.44367-4521.60465 D^{-1}
\end{aligned}
$$

A simpler method for $B A F=10$ is to multiply equations given in tables 1 to 12 by $\frac{1833.46495}{D^{2}}$ to obtain per-acre estimates. The per-acre total green weight for a 7-inch northern red oak, for example, is:

$$
\begin{aligned}
\text { W per acre } & =\left[\frac{1833.46495}{(7)^{2}}\right]\left[2.87249(7)^{2.66958}\right] \\
& =19382
\end{aligned}
$$

This procedure was used to produce tables 13 to 24 .

\section{Combined Equations for Red and White Oaks}

Data for the red oaks (northern red oak, black oak, and scarlet oak) and white oaks (white oak and chestnut oak) were combined to yield equations shown in Table 25. Foresters wishing to tally oaks in these two groups rather than by species will find these equations useful and can develop local or point sampling weight tables using the procedures previously discussed. 
Table 13

Total tree green weight per acre per in-tree $(B A F=10) . *$

\begin{tabular}{|c|c|c|c|c|c|c|c|c|c|}
\hline \multirow[b]{2}{*}{ Dbh (in.) } & \multicolumn{9}{|c|}{ Species } \\
\hline & NRO & BO & so & wo & $\mathrm{CO}$ & YP & $\mathbf{H}$ & BC & $\mathbf{R M}$ \\
\hline 2 & 8377 & 10058 & 11535 & 6294 & 9158 & 9848 & 8369 & 11788 & 9831 \\
\hline 3 & 10990 & 12260 & 13736 & 8520 & 11482 & 11541 & 10779 & 13727 & 12062 \\
\hline 4 & 13325 & 14110 & 15547 & 10562 & 13480 & 12915 & 12899 & 15294 & 13946 \\
\hline 5 & 15472 & 15734 & 17115 & 12478 & 15267 & 14093 & 14826 & 16631 & 15607 \\
\hline 6 & 17481 & 17199 & 18513 & 14299 & 16901 & 15134 & 16613 & 17810 & 17110 \\
\hline 7 & 19382 & 18544 & 19783 & 16044 & 18418 & 16075 & 18290 & 18871 & 18493 \\
\hline 8 & 21194 & 19793 & 20954 & 17726 & 19843 & 16936 & 19880 & 19842 & 19782 \\
\hline 9 & 22934 & 20965 & 22044 & 19357 & 21190 & 17735 & 21396 & 20739 & 20993 \\
\hline 10 & 24610 & 22072 & 23067 & 20942 & 22473 & 18481 & 22851 & 21577 & 22138 \\
\hline 11 & 26232 & 23123 & 24033 & 22487 & 23700 & 19183 & 24251 & 22363 & 23229 \\
\hline 12 & 27805 & 24127 & 24951 & 23997 & 24878 & 19847 & 25604 & 23106 & 24271 \\
\hline 13 & 29336 & 25088 & 25826 & 25476 & 26014 & 20478 & 26916 & 23811 & 25271 \\
\hline 14 & 30829 & 26013 & 26663 & 26926 & 27112 & 21080 & 28190 & 24483 & 26233 \\
\hline 15 & 32286 & 26904 & 27467 & 28350 & 28176 & 21656 & 29430 & 25126 & 27162 \\
\hline 16 & 33712 & 27766 & 28241 & 29750 & 29208 & 22210 & 30640 & 25743 & 28061 \\
\hline
\end{tabular}

*Weights in tables 13 to 24 are in pounds. 
Table 14

Total tree dry weight per acre per in-tree $(B A F=10)$.

\begin{tabular}{|c|c|c|c|c|c|c|c|c|c|}
\hline \multirow[b]{2}{*}{ Dbh (in.) } & \multicolumn{9}{|c|}{ Species } \\
\hline & NRO & BO & so & wo & $\mathrm{CO}$ & YP & $\mathbf{H}$ & $\mathrm{BC}$ & RM \\
\hline 2 & 4893 & 5575 & 6607 & 3842 & 5630 & 4135 & 5452 & 6373 & 4908 \\
\hline 3 & 6393 & 6837 & 7896 & 5105 & 6992 & 5096 & 7013 & 7572 & 6165 \\
\hline 4 & 7730 & 7901 & 8960 & 6246 & 8154 & 5910 & 8385 & 8557 & 7247 \\
\hline 5 & 8956 & 8840 & 9884 & 7304 & 9187 & 6631 & 9631 & 9409 & 8216 \\
\hline 6 & 10101 & 9689 & 10708 & 8299 & 10127 & 7284 & 10785 & 10168 & 9103 \\
\hline 7 & 11182 & 10470 & 11459 & 9246 & 10997 & 7886 & 11869 & 10857 & 9927 \\
\hline 8 & 12212 & 11198 & 12151 & 10154 & 11811 & 8448 & 12895 & 11491 & 10701 \\
\hline 9 & 13199 & 11881 & 12797 & 11027 & 12578 & 8976 & 13873 & 12082 & 11434 \\
\hline 10 & 14149 & 12528 & 13403 & 11873 & 13306 & 9477 & 14811 & 12635 & 12132 \\
\hline 11 & 15067 & 13143 & 13977 & 12693 & 14002 & 9954 & 15714 & 13158 & 12800 \\
\hline 12 & 15957 & 13731 & 14522 & 13491 & 14668 & 10411 & 16586 & 13654 & 13442 \\
\hline 13 & 16823 & 14295 & 15041 & 14270 & 15309 & 10849 & 17431 & 14127 & 14060 \\
\hline 14 & 17666 & 14838 & 15539 & 15031 & 15928 & 11271 & 18252 & 14579 & 14659 \\
\hline 15 & 18488 & 15362 & 16018 & 15775 & 16526 & 11679 & 19051 & 15013 & 15238 \\
\hline 16 & 19293 & 15869 & 16479 & 16506 & 17106 & 12074 & 19830 & 15431 & 15802 \\
\hline
\end{tabular}


Table 15

Total tree dry weight without bark per acre per in-tree (BAF=10).

\begin{tabular}{|c|c|c|c|c|c|c|c|c|c|}
\hline \multirow[b]{2}{*}{ Dbh (in.) } & \multicolumn{9}{|c|}{ Species } \\
\hline & NRO & BO & so & wo & $\mathrm{CO}$ & $\mathbf{Y P}$ & $\mathbf{H}$ & $\mathrm{BC}$ & $\mathbf{R M}$ \\
\hline 2 & 3881 & 4548 & 5254 & 3347 & 4453 & 3469 & 4489 & 5577 & 4277 \\
\hline 3 & 5157 & 5598 & 6399 & 4454 & 5615 & 4283 & 5877 & 6667 & 5386 \\
\hline 4 & 6311 & 6486 & 7358 & 5455 & 6620 & 4973 & 7115 & 7566 & 6343 \\
\hline 5 & 7380 & 7271 & 8201 & 6383 & 7522 & 5584 & 8253 & 8347 & 7201 \\
\hline 6 & 8387 & 7983 & 8961 & 7258 & 8349 & 6139 & 9316 & 9044 & 7988 \\
\hline 7 & 9345 & 8639 & 9658 & 8090 & 9119 & 6651 & 10321 & 9679 & 8720 \\
\hline 8 & 10262 & 9250 & 10305 & 8888 & 9843 & 7129 & 11278 & 10265 & 9408 \\
\hline 9 & 11146 & 9825 & 10912 & 9657 & 10529 & 7579 & 12196 & 10811 & 10060 \\
\hline 10 & 12001 & 10370 & 11485 & 10401 & 11184 & 8005 & 13081 & 11324 & 10681 \\
\hline 11 & 12831 & 10888 & 12029 & 11124 & 11811 & 8411 & 13936 & 11809 & 11276 \\
\hline 12 & 13639 & 11384 & 12549 & 11827 & 12414 & 8800 & 14766 & 12270 & 11848 \\
\hline 13 & 14426 & 11861 & 13046 & 12513 & 12995 & 9174 & 15572 & 12709 & 12399 \\
\hline 14 & 15196 & 12319 & 13525 & 13183 & 13558 & 9534 & 16358 & 13131 & 12933 \\
\hline 15 & 15950 & 12762 & 13986 & 13840 & 14104 & 9882 & 17126 & 13535 & 13451 \\
\hline 16 & 16688 & 13191 & 14431 & 14484 & 14635 & 10219 & 17876 & 13925 & 13953 \\
\hline
\end{tabular}


Table 16

Total tree dry weight of bark per acre per in-tree $(B A F=10)$.

\begin{tabular}{|c|c|c|c|c|c|c|c|c|c|}
\hline \multirow[b]{2}{*}{ Dbh (in.) } & \multicolumn{9}{|c|}{ Species } \\
\hline & NRO & BO & so & wo & $\mathrm{CO}$ & YP & H & $\mathrm{BC}$ & $\mathbf{R M}$ \\
\hline 2 & 1021 & 1024 & 1365 & 483 & 1160 & 714 & 991 & 801 & 630 \\
\hline 3 & 1228 & 1235 & 1484 & 638 & 1348 & 855 & 1124 & 905 & 776 \\
\hline 4 & 1400 & 1411 & 1574 & 777 & 1499 & 972 & 1230 & 988 & 900 \\
\hline 5 & 1549 & 1564 & 1648 & 906 & 1628 & 1074 & 1318 & 1056 & 1010 \\
\hline 6 & 1683 & 1701 & 1711 & 1026 & 1741 & 1166 & 1395 & 1116 & 1109 \\
\hline 7 & 1806 & 1827 & 1766 & 1141 & 1843 & 1249 & 1464 & 1170 & 1201 \\
\hline 8 & 1919 & 1943 & 1815 & 1250 & 1936 & 1325 & 1526 & 1218 & 1286 \\
\hline 9 & 2025 & 2052 & 1860 & 1355 & 2022 & 1397 & 1583 & 1262 & 1367 \\
\hline 10 & 2124 & 2154 & 1900 & 1457 & 2103 & 1464 & 1636 & 1303 & 1443 \\
\hline 11 & 2218 & 2251 & 1938 & 1555 & 2178 & 1528 & 1686 & 1341 & 1516 \\
\hline 12 & 2308 & 2343 & 1973 & 1651 & 2249 & 1588 & 1732 & 1376 & 1585 \\
\hline 13 & 2394 & 2431 & 2005 & 1744 & 2317 & 1646 & 1776 & 1410 & 1652 \\
\hline 14 & 2476 & 2516 & 2036 & 1835 & 2381 & 1702 & 1817 & 1442 & 1716 \\
\hline 15 & 2555 & 2598 & 2065 & 1924 & 2442 & 1755 & 1857 & 1472 & 1779 \\
\hline 16 & 2631 & 2676 & 2093 & 2011 & 2501 & 1806 & 1895 & 1501 & 1839 \\
\hline
\end{tabular}


Table 17

Green weight of branches per acre per in-tree (BAF=10).

\begin{tabular}{|c|c|c|c|c|c|c|c|c|c|}
\hline \multirow[b]{2}{*}{ Dbh (in.) } & \multicolumn{9}{|c|}{ Species } \\
\hline & NRO & BO & so & wo & co & YP & $\mathbf{H}$ & BC & $\mathbf{R M}$ \\
\hline 5 & 7884 & 4770 & 6836 & 5149 & 5797 & 5345 & 5759 & 5330 & 6855 \\
\hline 6 & 6790 & 4927 & 5457 & 3928 & 4617 & 2663 & 4453 & 4531 & 6121 \\
\hline 7 & 6298 & 5063 & 5003 & 3529 & 4247 & 1626 & 4194 & 4108 & 5646 \\
\hline 8 & 6088 & 5183 & 4952 & 3490 & 4228 & 1328 & 4369 & 3871 & 5318 \\
\hline 9 & 6018 & 5292 & 5085 & 3613 & 4367 & 1381 & 4724 & 3735 & 5079 \\
\hline 10 & 6021 & 5392 & 5300 & 3808 & 4574 & 1603 & 5145 & 3656 & 4897 \\
\hline 11 & 6063 & 5483 & 5547 & 4032 & 4808 & 1903 & 5581 & 3612 & 4756 \\
\hline 12 & 6124 & 5568 & 5802 & 4262 & 5047 & 2235 & 6007 & 3588 & 4642 \\
\hline 13 & 6196 & 5648 & 6053 & 4489 & 5280 & 2573 & 6411 & 3578 & 4550 \\
\hline 14 & 6271 & 5722 & 6294 & 4705 & 5502 & 2906 & 6791 & 3577 & 4473 \\
\hline 15 & 6346 & 5792 & 6521 & 4910 & 5712 & 3225 & 7144 & 3581 & 4408 \\
\hline 16 & 6420 & 5858 & 6735 & 5102 & 5908 & 3529 & 7471 & 3588 & 4353 \\
\hline
\end{tabular}


Table 18

Dry weight of branches per acre per in-tree (BAF=10).

\begin{tabular}{|c|c|c|c|c|c|c|c|c|c|}
\hline \multirow[b]{2}{*}{ Dbh (in.) } & \multicolumn{9}{|c|}{ Species } \\
\hline & NRO & BO & so & wo & Co & YP & $\mathbf{H}$ & BC & $\mathbf{R M}$ \\
\hline 5 & 4565 & 3537 & 4233 & 3367 & 3278 & 2438 & 3694 & 2867 & 3582 \\
\hline 6 & 3967 & 2960 & 3253 & 2293 & 2612 & 1305 & 2771 & 2511 & 3277 \\
\hline 7 & 3706 & 2802 & 2932 & 1908 & 2417 & 860 & 2588 & 2307 & 3058 \\
\hline 8 & 3601 & 2823 & 2898 & 1828 & 2423 & 725 & 2712 & 2182 & 2894 \\
\hline 9 & 3573 & 2921 & 2995 & 1890 & 2519 & 739 & 2963 & 2102 & 2765 \\
\hline 10 & 3585 & 3051 & 3150 & 2017 & 2653 & 825 & 3261 & 2048 & 2661 \\
\hline 11 & 3617 & 3192 & 3329 & 2173 & 2800 & 944 & 3569 & 2010 & 2577 \\
\hline 12 & 3659 & 3333 & 3512 & 2338 & 2949 & 1078 & 3870 & 1984 & 2506 \\
\hline 13 & 3706 & 3469 & 3693 & 2504 & 3093 & 1214 & 4156 & 1965 & 2446 \\
\hline 14 & 3754 & 3597 & 3865 & 2663 & 3230 & 1349 & 4425 & 1952 & 2395 \\
\hline 15 & 3801 & 3718 & 4029 & 2816 & 3359 & 1479 & 4674 & 1941 & 2350 \\
\hline 16 & 3847 & 3831 & 4182 & 2959 & 3480 & 1603 & 4906 & 1934 & 2311 \\
\hline
\end{tabular}


Table 19

Dry weight of branches without bark per acre per in-tree (BAF=10).

\begin{tabular}{|c|c|c|c|c|c|c|c|c|c|}
\hline \multirow[b]{2}{*}{ Dbh (in.) } & \multicolumn{9}{|c|}{ Species } \\
\hline & NRO & BO & so & wo & Co & YP & H & BC & $\mathbf{R M}$ \\
\hline 5 & 3601 & 2830 & 3441 & 2853 & 2540 & 2146 & 3002 & 2489 & 3117 \\
\hline 6 & 3225 & 2346 & 2688 & 1898 & 2117 & 1099 & 2234 & 2189 & 2821 \\
\hline 7 & 3053 & 2214 & 2445 & 1541 & 1995 & 683 & 2109 & 2016 & 2611 \\
\hline 8 & 2979 & 2231 & 2424 & 1450 & 2002 & 552 & 2247 & 1909 & 2454 \\
\hline 9 & 2952 & 2313 & 2504 & 1484 & 2066 & 557 & 2492 & 1839 & 2332 \\
\hline 10 & 2951 & 2422 & 2627 & 1578 & 2154 & 629 & 2774 & 1791 & 2235 \\
\hline 11 & 2963 & 2540 & 2769 & 1698 & 2250 & 733 & 3061 & 1758 & 2155 \\
\hline 12 & 2983 & 2659 & 2913 & 1828 & 2347 & 850 & 3341 & 1734 & 2089 \\
\hline 13 & 3006 & 2773 & 3055 & 1960 & 2441 & 971 & 3605 & 1716 & 2033 \\
\hline 14 & 3030 & 2881 & 3191 & 2089 & 2530 & 1090 & 3852 & 1703 & 1986 \\
\hline 15 & 3055 & 2982 & 3320 & 2212 & 2614 & 1206 & 4081 & 1693 & 1944 \\
\hline 16 & 3079 & 3076 & 3440 & 2328 & 2692 & 1316 & 4293 & 1686 & 1908 \\
\hline
\end{tabular}


Table 20

Dry weight of branch bark per acre per in-tree $(B A F=10)$.

\begin{tabular}{|c|c|c|c|c|c|c|c|c|c|}
\hline \multirow[b]{2}{*}{ Dbh (in.) } & \multicolumn{9}{|c|}{ Species } \\
\hline & NRO & BO & so & wo & $\mathrm{CO}$ & YP & $\mathbf{H}$ & $\mathrm{BC}$ & RM \\
\hline 5 & 964 & 707 & 793 & 513 & 740 & 225 & 686 & 378 & 465 \\
\hline 6 & 743 & 614 & 565 & 394 & 495 & 222 & 625 & 322 & 456 \\
\hline 7 & 653 & 588 & 486 & 367 & 420 & 219 & 578 & 291 & 447 \\
\hline 8 & 622 & 592 & 474 & 378 & 420 & 216 & 540 & 273 & 439 \\
\hline 9 & 621 & 608 & 492 & 406 & 451 & 214 & 509 & 263 & 433 \\
\hline 10 & 634 & 629 & 523 & 440 & 498 & 212 & 482 & 257 & 427 \\
\hline 11 & 654 & 651 & 560 & 475 & 549 & 211 & 459 & 253 & 421 \\
\hline 12 & 676 & 674 & 599 & 510 & 601 & 209 & 440 & 250 & 417 \\
\hline 13 & 700 & 696 & 637 & 543 & 651 & 208 & 422 & 249 & 413 \\
\hline 14 & 724 & 717 & 674 & 575 & 700 & 207 & 406 & 248 & 409 \\
\hline 15 & 747 & 736 & 709 & 604 & 745 & 205 & 392 & 248 & 406 \\
\hline 16 & 769 & 754 & 742 & 631 & 788 & 204 & 380 & 248 & 403 \\
\hline
\end{tabular}


Table 21

Green weight to a 4-inch dob per acre per in-tree (BAF=10).

\begin{tabular}{|c|c|c|c|c|c|c|c|c|c|}
\hline \multirow[b]{2}{*}{ Dbh (in.) } & \multicolumn{9}{|c|}{ Species } \\
\hline & NRO & BO & so & wo & $\mathrm{CO}$ & YP & $\mathbf{H}$ & BC & RM \\
\hline 5 & 11911 & 16302 & 13513 & 8498 & 10551 & 8894 & 6775 & 12132 & 14921 \\
\hline 6 & 13886 & 14249 & 14585 & 12004 & 13138 & 11237 & 8579 & 13463 & 12173 \\
\hline 7 & 15517 & 14025 & 15620 & 14361 & 14977 & 13042 & 10475 & 14820 & 12190 \\
\hline 8 & 16861 & 14536 & 16545 & 16049 & 16351 & 14468 & 12452 & 16060 & 13287 \\
\hline 9 & 17978 & 15335 & 17351 & 17313 & 17417 & 15620 & 14503 & 17155 & 14781 \\
\hline 10 & 18916 & 16229 & 18052 & 18295 & 18268 & 16568 & 16623 & 18115 & 16382 \\
\hline 11 & 19713 & 17127 & 18661 & 19079 & 18964 & 17362 & 18806 & 18954 & 17958 \\
\hline 12 & 20398 & 17991 & 19194 & 19718 & 19542 & 18035 & 21049 & 19692 & 19456 \\
\hline 13 & 20992 & 18804 & 19663 & 20249 & 20031 & 18614 & 23348 & 20343 & 20854 \\
\hline 14 & 21511 & 19561 & 20078 & 20697 & 20449 & 19116 & 25699 & 20920 & 22147 \\
\hline 15 & 21970 & 20261 & 20447 & 21080 & 20812 & 19557 & 28101 & 21435 & 23339 \\
\hline 16 & 22377 & 20907 & 20777 & 21412 & 21129 & 19945 & 30550 & 21897 & 24436 \\
\hline
\end{tabular}


Table 22

Dry weight to a 4-inch dob per acre per in-tree $(B A F=10)$.

\begin{tabular}{|c|c|c|c|c|c|c|c|c|c|}
\hline \multirow[b]{2}{*}{ Dbh (in.) } & \multicolumn{9}{|c|}{ Species } \\
\hline & NRO & BO & so & wo & Co & YP & $\mathrm{H}$ & $\mathrm{BC}$ & RM \\
\hline 5 & 6689 & 9106 & 6445 & 6264 & 7287 & 4722 & 4448 & 6848 & 7723 \\
\hline 6 & 7930 & 7923 & 7211 & 7023 & 8015 & 5812 & 5619 & 8216 & 6564 \\
\hline 7 & 8900 & 7810 & 7930 & 7737 & 8688 & 6723 & 6846 & 9185 & 6741 \\
\hline 8 & 9673 & 8124 & 8609 & 8414 & 9316 & 7478 & 8124 & 9907 & 7422 \\
\hline 9 & 10300 & 8606 & 9257 & 9060 & 9907 & 8109 & 9448 & 10466 & 8277 \\
\hline 10 & 10819 & 9140 & 9878 & 9679 & 10468 & 8641 & 10814 & 10912 & 9166 \\
\hline 11 & 11255 & 9676 & 10475 & 10276 & 11002 & 9094 & 12219 & 11275 & 10029 \\
\hline 12 & 11625 & 10191 & 11052 & 10854 & 11514 & 9484 & 13660 & 11577 & 10842 \\
\hline 13 & 11945 & 10674 & 11611 & 11413 & 12006 & 9823 & 15136 & 11832 & 11595 \\
\hline 14 & 12222 & 11124 & 12153 & 11957 & 12480 & 10120 & 16644 & 12050 & 12289 \\
\hline 15 & 12466 & 11540 & 12681 & 12486 & 12938 & 10382 & 18183 & 12238 & 12927 \\
\hline 16 & 12681 & 11924 & 13195 & 13002 & 13382 & 10614 & 19751 & 12403 & 13512 \\
\hline
\end{tabular}


Table 23

Dry weight without bark to a 4-inch dob per acre per in-tree (BAF=10).

\begin{tabular}{|c|c|c|c|c|c|c|c|c|c|}
\hline \multirow[b]{2}{*}{ Dbh (in.) } & \multicolumn{9}{|c|}{ Species } \\
\hline & NRO & BO & so & wo & co & YP & H & $\mathrm{BC}$ & RM \\
\hline $\begin{array}{l}5 \\
6\end{array}$ & $\begin{array}{l}5454 \\
6748\end{array}$ & $\begin{array}{l}7426 \\
6577\end{array}$ & $\begin{array}{l}5471 \\
6157\end{array}$ & $\begin{array}{l}5753 \\
6412\end{array}$ & $\begin{array}{l}6372 \\
7052\end{array}$ & $\begin{array}{l}4059 \\
4796\end{array}$ & $\begin{array}{l}3724 \\
4784\end{array}$ & $\begin{array}{l}6089 \\
7287\end{array}$ & $\begin{array}{l}6687 \\
5823\end{array}$ \\
\hline 7 & 7689 & 6549 & 6804 & 7029 & 7697 & 5523 & 5913 & 8170 & 6046 \\
\hline 8 & 8404 & 6844 & 7418 & 7611 & 8269 & 6180 & 7105 & 8848 & 6672 \\
\hline 9 & 8966 & 7261 & 8007 & 8164 & 8765 & 6755 & 8353 & 9384 & 7432 \\
\hline 10 & 9418 & 7712 & 8572 & 8693 & 9195 & 7257 & 9654 & 9818 & 8211 \\
\hline 11 & 9790 & 8160 & 9118 & 9201 & 9568 & 7695 & 11006 & 10177 & 8962 \\
\hline 12 & 10102 & 8587 & 9647 & 9690 & 9894 & 8078 & 12404 & 10479 & 9665 \\
\hline 13 & 10367 & 8986 & 10161 & 10163 & 10180 & 8416 & 13846 & 10737 & 10316 \\
\hline 14 & 10595 & 9356 & 10660 & 10622 & 10433 & 8715 & 15330 & 10958 & 10915 \\
\hline 15 & 10793 & 9697 & 11148 & 11068 & 10658 & 8982 & 16855 & 11152 & 11463 \\
\hline 16 & 10967 & 10011 & 11624 & 11501 & 10859 & 9220 & 18418 & 11322 & 11966 \\
\hline
\end{tabular}


Table 24

Dry weight of bark to a 4-inch dob per acre per in-tree (BAF=10).

\begin{tabular}{|c|c|c|c|c|c|c|c|c|c|}
\hline \multirow[b]{2}{*}{ Dbh (in.) } & \multicolumn{9}{|c|}{ Species } \\
\hline & NRO & BO & so & wo & co & YP & H & BC & RM \\
\hline 5 & 1235 & 1680 & 971 & 509 & 1135 & 663 & 696 & 758 & 1027 \\
\hline 6 & 1182 & 1346 & 1047 & 604 & 1226 & 1016 & 784 & 929 & 738 \\
\hline 7 & 1211 & 1261 & 1115 & 698 & 1308 & 1199 & 868 & 1015 & 694 \\
\hline 8 & 1268 & 1280 & 1179 & 791 & 1384 & 1299 & 947 & 1059 & 750 \\
\hline 9 & 1334 & 1345 & 1237 & 884 & 1454 & 1354 & 1023 & 1082 & 847 \\
\hline 10 & 1401 & 1428 & 1292 & 975 & 1520 & 1384 & 1097 & 1093 & 958 \\
\hline 11 & 1464 & 1516 & 1344 & 1067 & 1582 & 1400 & 1167 & 1097 & 1070 \\
\hline 12 & 1523 & 1604 & 1393 & 1157 & 1641 & 1406 & 1236 & 1097 & 1179 \\
\hline 13 & 1577 & 1689 & 1440 & 1248 & 1697 & 1407 & 1303 & 1095 & 1282 \\
\hline 14 & 1627 & 1769 & 1485 & 1337 & 1751 & 1405 & 1368 & 1091 & 1378 \\
\hline 15 & 1672 & 1843 & 1528 & 1427 & 1802 & 1400 & 1431 & 1087 & 1467 \\
\hline 16 & 1714 & 1913 & 1569 & 1516 & 1852 & 1394 & 1493 & 1082 & 1550 \\
\hline
\end{tabular}


Table 25

Weight equations for the red oak and white oak groups.

\begin{tabular}{|c|c|c|c|c|c|}
\hline \multirow[b]{2}{*}{ Weight } & \multirow[b]{2}{*}{ Group } & \multirow[b]{2}{*}{ Equation } & \multirow[b]{2}{*}{$\mathbf{R}^{2}$} & \multicolumn{2}{|c|}{ Standard error } \\
\hline & & & & Pounds & $\%$ of $\mathrm{W}$-mean \\
\hline Total tree green wt. & $\begin{array}{l}\text { Red oaks } \\
\text { White oaks }\end{array}$ & $\begin{array}{l}W=3.64938 D^{2.54036} \\
W=2.68231 D^{2.64500}\end{array}$ & $\begin{array}{l}.990 \\
.987\end{array}$ & $\begin{array}{l}109 \\
130\end{array}$ & $\begin{array}{r}9 \\
10\end{array}$ \\
\hline Total tree dry wt. & $\begin{array}{l}\text { Red oaks } \\
\text { White oaks }\end{array}$ & $\begin{array}{l}W=2.09014 D^{2.54052} \\
W=1.68565 D^{2.60982}\end{array}$ & $\begin{array}{l}.989 \\
.985\end{array}$ & $\begin{array}{l}69 \\
79\end{array}$ & $\begin{array}{l}10 \\
11\end{array}$ \\
\hline $\begin{array}{l}\text { Total tree dry wt. } \\
\text { without bark }\end{array}$ & $\begin{array}{l}\text { Red oaks } \\
\text { White oaks }\end{array}$ & $\begin{array}{l}W=1.63495 D^{2.57352} \\
W=1.37211 D^{2.63256}\end{array}$ & $\begin{array}{l}.989 \\
.986\end{array}$ & $\begin{array}{l}61 \\
66\end{array}$ & $\begin{array}{l}10 \\
11\end{array}$ \\
\hline $\begin{array}{l}\text { Total tree dry wt. } \\
\text { of bark }\end{array}$ & $\begin{array}{l}\text { Red oaks } \\
\text { White oaks }\end{array}$ & $\begin{array}{l}W=0.46923 D^{2.37764} \\
W=0.29702 D^{2.50850}\end{array}$ & $\begin{array}{l}.985 \\
.951\end{array}$ & $\begin{array}{l}12 \\
21\end{array}$ & $\begin{array}{l}11 \\
20\end{array}$ \\
\hline Green wt. of branches & $\begin{array}{l}\text { Red oaks } \\
\text { White oaks }\end{array}$ & $\begin{array}{l}W=167.47727+5.57660 D^{2}-42.43430 D \\
W=184.52253+5.32224 D^{2}-48.73647 D\end{array}$ & $\begin{array}{l}.897 \\
.939\end{array}$ & $\begin{array}{l}86 \\
55\end{array}$ & $\begin{array}{l}24 \\
19\end{array}$ \\
\hline Dry wt. of branches & $\begin{array}{l}\text { Red oaks } \\
\text { White oaks }\end{array}$ & $\begin{array}{l}W=114.40758+3.55460 D^{2}-29.31907 D \\
W=124.89440+3.31160 D^{2}-32.77917 D\end{array}$ & $\begin{array}{l}.891 \\
.910\end{array}$ & $\begin{array}{l}53 \\
40\end{array}$ & $\begin{array}{l}25 \\
23\end{array}$ \\
\hline $\begin{array}{l}\text { Dry wt. of branches } \\
\text { without bark }\end{array}$ & $\begin{array}{l}\text { Red oaks } \\
\text { White oaks }\end{array}$ & $\begin{array}{l}W=88.24854+2.83470 D^{2}-22.75065 D \\
W=93.46358+2.51626 D^{2}-24.25798 D\end{array}$ & $\begin{array}{l}.871 \\
.902\end{array}$ & $\begin{array}{l}48 \\
32\end{array}$ & $\begin{array}{l}27 \\
24\end{array}$ \\
\hline Dry wt. of branch bark & $\begin{array}{l}\text { Red oaks } \\
\text { White oaks }\end{array}$ & $\begin{array}{l}W=26.15905+0.71990 D^{2}-6.56843 D \\
W=31.55046+0.79647 D^{2}-8.54744 D\end{array}$ & $\begin{array}{l}.902 \\
.893\end{array}$ & $\begin{array}{l}10 \\
10\end{array}$ & $\begin{array}{l}24 \\
27\end{array}$ \\
\hline $\begin{array}{l}\text { Green wt. to a } 4 \text {-inch } \\
\text { dob }\end{array}$ & $\begin{array}{l}\text { Red oaks } \\
\text { White oaks }\end{array}$ & $\begin{array}{l}W=208.76407+16.11594 D^{2}-85.39248 D \\
W=21.03646+14.57852 D^{2}-48.52150 D\end{array}$ & $\begin{array}{l}.978 \\
.984\end{array}$ & $\begin{array}{l}127 \\
111\end{array}$ & $\begin{array}{r}11 \\
9\end{array}$ \\
\hline $\begin{array}{l}\text { Dry wt. to a 4-inch } \\
\text { dob }\end{array}$ & $\begin{array}{l}\text { Red oaks } \\
\text { White oaks }\end{array}$ & $\begin{array}{l}W=80.61690+8.82363 D^{2}-40.83982 D \\
W=1.50659 D^{2.56224}\end{array}$ & $\begin{array}{l}.975 \\
.983\end{array}$ & $\begin{array}{l}79 \\
66\end{array}$ & $\begin{array}{l}12 \\
10\end{array}$ \\
\hline $\begin{array}{l}\text { Dry wt. without bark } \\
\text { to a 4-inch dob }\end{array}$ & $\begin{array}{l}\text { Red oaks } \\
\text { White oaks }\end{array}$ & $\begin{array}{l}W=67.75124+7.65651 D^{2}-35.67783 D \\
W=1.32023 D^{2.56152}\end{array}$ & $\begin{array}{l}.970 \\
.985\end{array}$ & $\begin{array}{l}74 \\
54\end{array}$ & $\begin{array}{r}13 \\
9\end{array}$ \\
\hline $\begin{array}{l}\text { Dry wt. of bark } \\
\text { to a } 4 \text {-inch dob }\end{array}$ & $\begin{array}{l}\text { Red oaks } \\
\text { White oaks }\end{array}$ & $\begin{array}{l}W=0.23620 D^{2.50780} \\
W=0.16386 D^{2.61120}\end{array}$ & $\begin{array}{l}.937 \\
.908\end{array}$ & $\begin{array}{l}17 \\
21\end{array}$ & $\begin{array}{l}19 \\
24\end{array}$ \\
\hline
\end{tabular}




\section{SELECTED REFERENCES}

Belanger, R. P. 1973. Volume and weight tables for plantation-grown sycamore. USDA For. Ser. Res. Paper SE-107.

Burkhart, H. E., and J. L. Clutler. 1971. Green and dry weight yields for old field loblolly pine plantations in the Georgia piedmont. Ga. For. Res. Council Report 22.

Curtis, F. H. 1965. Tree weight equations-their development and use in forest management planning. Soc. Amer. For. Proc. pp. 189-191.

Kulow, D. L. 1965. Elementary point-sampling. W. Va. Univ. Agr. Exp. Sta. Cir. 116.

Lenhart, J. D., J. R. Hasness, D. R. Hicks, D. M. Hyink, and S. I. Somberg. 1973. Estimating cubic foot volume, green weight, or dry weight per acre of planted loblolly pine using variable-radius-plot cruising techniques. Stephen F. Austin State Univ. Texas Forest. Paper 21.

Ribe, J. H. 1973. Puckerbrush weight tables. Univ. Maine Life Sci. \& Agr. Exp. Sta. Misc. Report 152.

Schnur, G. L. 1937. Yield, stand, and volume tables for even-aged upland oak forests. USDA Tech Bul. 560.

Young, H. E. 1976. A summary and analysis of weight tables studies. Complete Tree Institute, Univ. of Maine.

Wartluft, J. L. 1977. Weights of small Appalachian hardwood trees and components. USDA For. Ser. Res. Paper NE-366.

Wiant, H. V., Jr. 1976. Elementary 3P sampling. W. Va. Univ. Agr. \& Forest. Exp. Sta. Bul. 650T. 
Blank Page in Original Bulletin 
Blank Page in Original Bulletin 